\title{
D-branes in VSFT and their low energy limit
}

\author{
L.Bonora*, D.Mamone, M.Salizzoni \\ International School for Advanced Studies (SISSA/ISAS) \\ Via Beirut 2-4, 34014 Trieste, Italy, and INFN, Sezione di Trieste \\ E-mail: bonora@sissa.it, mamone@sissa.it, 'sali@sissa.it!
}

ABSTRACT: This contribution is devoted to a comparison between solitonic solutions in noncommutative field theories and tachyonic lumps in vacuum string field theory. We analyze in detail a sequence of the latter in the presence of a constant background $B$ field. We show, in particular, that there exists a remarkable isomorphism with the sequence of so-called GMS solitons in noncommutative field theories.

\section{Introduction}

This contribution is devoted to a comparison between classical solutions in vacuum string field theory (VSFT) and in scalar noncommutative field theories. In particular it is possible to establish a one-to-one correspondence between tachyonic lumps, i.e lower dimensional D-branes, in the former and solitonic solutions in the latter. This correspondence is fully exposed by introducing a constant background $B$ field.

\section{VSFT}

VSFT is a version of Witten's open SFT [i] corresponding to the minimum of the tachyon potential. Witten's string field theory action is

$$
\mathcal{S}(\Psi)=-\frac{1}{g_{0}^{2}}\left[\frac{1}{2}\langle\Psi, Q \Psi\rangle+\frac{1}{3}\langle\Psi, \Psi * \Psi\rangle\right]
$$

This action represents open string theory about the trivial unstable vacuum $\left|\Psi_{0}\right\rangle=c_{1}|0\rangle$. It was argued by A.Sen, [2] $]$, that at the minimum of the tachyon potential a dramatic change occurs in this theory, so that corresponding to the new vacuum it represents closed string theory rather that the open string theory we started with. In particular, this theory is expected to host tachyonic lumps representing unstable D-branes of any dimension less than 25. Unfortunately we have been so far unable to find an exact classical solution, say $\left|\Phi_{0}\right\rangle$, representing the new vacuum. One can nevertheless guess the form taken by the

\footnotetext{
${ }^{*}$ Speaker.
} 


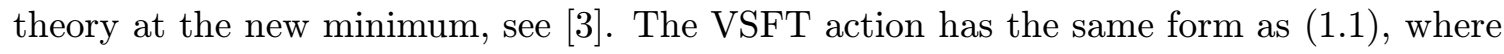
the new string field is still denoted by $\Psi$, the ${ }^{*}$ product is the same as in the previous theory, while with the BRST operator $Q$ is replaced by a new one, usually denoted $\mathcal{Q}$, which is characterized by universality and vanishing cohomology. In the Siegel gauge,

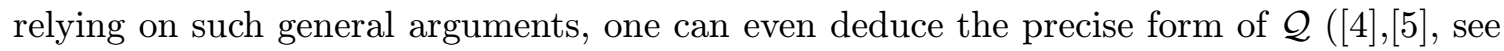

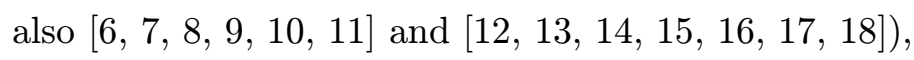

$$
\mathcal{Q}=c_{0}+(-1)^{n} \sum_{n>0}\left(c_{2 n}+c_{-2 n}\right)
$$

Now, the equation of motion of VSFT is

$$
\mathcal{Q} \Psi=-\Psi * \Psi
$$

and nonperturbative solutions are looked for in the form

$$
\Psi=\Psi_{m} \otimes \Psi_{g}
$$

where $\Psi_{g}$ and $\Psi_{m}$ depend purely on ghost and matter degrees of freedom, respectively.

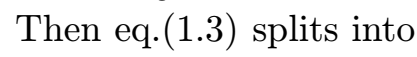

$$
\begin{aligned}
\mathcal{Q} \Psi_{g} & =-\Psi_{g} * \Psi_{g} \\
\Psi_{m} & =\Psi_{m} * \Psi_{m}
\end{aligned}
$$

We can assume that the solution for the ghost part is calculated once and for all (this

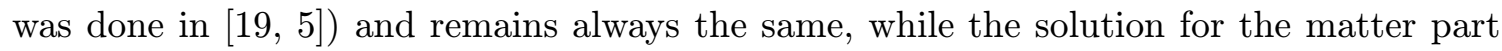
varies. Therefore Eq.(1). (1) will not be involved in our analysis and we will concentrate on

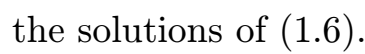

The value of the action for such solutions is given by

$$
\mathcal{S}(\Psi)=\mathcal{K}\left\langle\Psi_{m} \mid \Psi_{m}\right\rangle
$$

where $\mathcal{K}$ contains the ghost contribution. It must be remarked, as shown in [1, 12$], \mathcal{K}$ is infinite unless the action is suitably regularized. The choice of a regularization should be understood as a 'gauge' freedom in choosing the solutions of (1 $1 . \overline{3})$, so that a coupled solution of (1) (1) and (1) (1) nevertheless a legitimate representative of the corresponding class of solutions.

\section{Solitons in noncommutative field theories}

After this short introduction to VSFT, let us now briefly describe solitons in noncommutative field theories, (see $[\overline{2} \overline{0} \overline{0}]$ for a beautiful review). Noncommutative field theories are effective low energy field theories which live on the world-volume of D-branes in the presence of a constant background $B$ field. To be definite, let us think of a D25-brane in bosonic string theory. The simplest example of effective theories is a noncommutative theory of a scalar field $\phi$, which is thought to represent the tachyon living on the brane (this is an oversimplified situation, in fact one could easily take the gauge field as well into 
account, while the massive string states are thought to having been integrated out). We concentrate on the case in which $B$ is switched on along two space directions, which we denote by $x=q$ and $y=p$ (from now one, for simplicity, we will drop the other coordinates). The coordinates become noncommutative and the ensuing situation can be described by replacing the initial theory with a theory in which all products are replaced by the Moyal $\star$ product with deformation parameter $\theta$ (linked to $B$ as explained below). Alternatively one can use the Weyl map and replace the noncommutative coordinates by two conjugate operators $\hat{q}, \hat{p}$, such that $[\hat{q}, \hat{p}]=i \theta$. In the large $\theta$ limit, after a suitable rescaling of the coordinates, the kinetic part of the action becomes negligible, so only the potential part, $\int d x d y V_{\star}(\phi)$, is relevant. Using the Weyl correspondence, the action can be replaced by $2 \pi \theta \operatorname{Tr}_{\mathcal{H}} V(\hat{\phi})$, where $\mathcal{H}$ is the Hilbert space constructed out of $\hat{q}, \hat{p}$, and $\hat{\phi}$ is the operator corresponding to the noncommutative field $\phi$. Solutions to the equations of motion take the form

$$
\hat{\phi}=\lambda_{i} P, \quad P^{2}=P
$$

where $\lambda_{i}, i=1, \ldots, n$ are the minima of the classical commutative potential $V$, which is assumed to be polynomial. The energy of such a solution is therefore given by $2 \pi \theta V\left(\lambda_{i}\right) \operatorname{Tr}_{\mathcal{H}} P$.

On the basis of this discussion it is clear that, in order to know the finite energy solutions of the noncommutative scalar theory, we have to find the finite rank projectors in the space $\mathcal{H}$. The latter can be constructed in the following way. Define the harmonic oscillator $a=(\hat{q}+i \hat{p}) / \sqrt{(2 \theta)}$ and its hermitean conjugate $a^{\dagger}:\left[a, a^{\dagger}\right]=1$. By a standard construction we can define the normalized harmonic oscillator eigenstates: $|n\rangle=\frac{\left(a^{\dagger}\right)^{n}}{\sqrt{n} !}|0\rangle$. Now, via the Weyl correspondence, we can map any operator $|n\rangle\langle m|$ to a classical function of the coordinates $x, y$. In particular $|n\rangle\langle n|$, which are rank one projectors, will be mapped to classical functions

$$
\psi_{n}(x, y)=2(-1)^{n} L_{n}\left(\frac{2 r^{2}}{\theta}\right) e^{-\frac{r^{2}}{\theta}}
$$

where $r^{2}=x^{2}+y^{2}$. Each of these solutions, by construction, satisfy $\psi_{n} \star \psi_{n}=\psi_{n}$. We refer to them as the GMS solitons. They can be interpreted as D23-branes. We can of course consider any finite sum of these projectors. They will also be solutions. They are interpreted as collapsing D23-branes. Moreover, using the shift operator $S=$ $\sum_{n=0}^{\infty}|n+1\rangle\langle n|$, one can set up a solution generating techniques, whereby a nontrivial soliton solution can be generated starting from a trivial one by repeated application of $S$

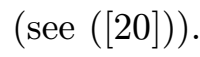

Our purpose in this paper is to show how the soliton solutions just described arise in VSFT. The way they can be seen is by taking the low energy limit of tachyonic lumps

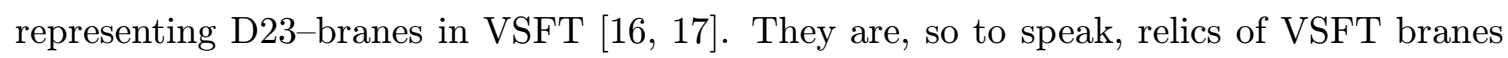
in the $\alpha^{\prime} \rightarrow 0$ limit.

\section{The background $B$ field}

But before we turn to this, we will introduce a background field $B$ in SFT. In ref.

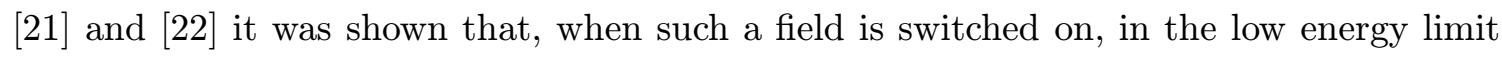


the string field theory star product factorizes into the ordinary Witten $*$ product and the Moyal $\star$ product. A related result can be obtained in the following way. The string field theory action ( $\left.\overline{1}_{1} \overline{1}_{1}^{\prime}\right)$ can be explicitly calculated in terms of local fields, provided the string field is expressed itself in terms of local fields

$$
|\Psi\rangle=\left(\phi(x)+A_{\mu}(x) a_{1}^{\mu \dagger}+\ldots\right) c_{1}|0\rangle
$$

Of course this makes sense in the limit in which string theory can be approximated by a local field theory. In this framework (1) (1) the form of an infinite series of integrated local polynomials (kinetic and potential terms) of the fields involved in (i.10). Now, it

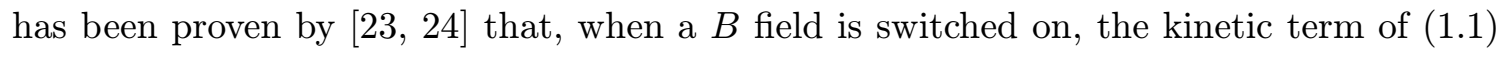
remains the same while the three string vertex changes, being multiplied by a (cyclically invariant) noncommutative phase factor (see $(\overline{2} \overline{2} \overline{3}, \overline{2} \overline{2} \overline{4}])$ and eq. $(\overline{2} \cdot \overline{2} \cdot \bar{i})$ below). It is easy to see on a general basis that the overall effect of such noncommutative factor is to replace the ordinary product with the Moyal product in the RHS of the effective action (for a related

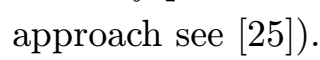

Therefore, we know pretty well the effects of a $B$ field when perturbative configurations are involved. What we wish to explore here are the effects of a $B$ field on nonperturbative solutions. We find that a $B$ field has the virtue of smoothing out some of the singularities that appear in VSFT. As for the overall star product in the presence of a background $B$ field, it turns out that Witten's star product and Moyal product are completely entangled in nonperturbative configurations. Nevertheless, in the low energy limit, we can again witness the factorization into Witten's star product and the Moyal one. It is exactly this factorization that allows us to recover the noncommutative field theory solitons.

We also remark that switching on a $B$ field in VSFT is consistent with the interpretation of VSFT. The latter is thought to describe closed string theory, and the antisymmetric field $B$ belongs in the massless sector of a bosonic closed string theory.

Finally we would like to stress that the Moyal star product referred to here has nothing to do with the Moyal representation of Witten's star product which was suggested in

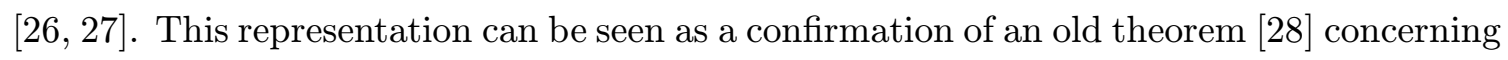
the uniqueness of the Moyal product in the class of noncommutative associative products,

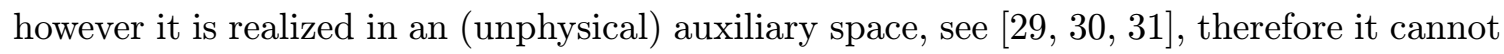
affect the physical space-time.

\section{Organization of the paper}

This contribution is organized as follows. In section 2 we derive the new Neumann coefficients for the three string vertex in the presence of a background $B$ field. In section 3 we solve the projector equation (1.06) for a 23-dimensional tachyonic lump and justify its D23-brane interpretation. In section 4 we start examining the effect of the $B$ field on such solution. In section 5 we generalize the lump solution of section 3 . We construct a series of solutions of the matter projector equation, which we denote by $\left|\Lambda_{n}\right\rangle$ for any natural number $n .\left|\Lambda_{n}\right\rangle$ is generated by acting on a tachyonic lump solution $\left|\Lambda_{0}\right\rangle$ with $(-\kappa)^{n} L_{n}(\mathbf{x} / \kappa)$, where $L_{n}$ is the $n$-th Laguerre polynomial, $\mathbf{x}$ is a quadratic expression in 
the string creation operators, see below eqs.(15.6.5. These states satisfy the remarkable properties

$$
\begin{aligned}
& \left|\Lambda_{n}\right\rangle *\left|\Lambda_{m}\right\rangle=\delta_{n, m}\left|\Lambda_{n}\right\rangle \\
& \left\langle\Lambda_{n} \mid \Lambda_{m}\right\rangle=\delta_{n, m}\left\langle\Lambda_{0} \mid \Lambda_{0}\right\rangle
\end{aligned}
$$

Each $\left|\Lambda_{n}\right\rangle$ represents a D23-brane, parallel to all the others. In section 6 we show that the field theory limit of $\left|\Lambda_{n}\right\rangle$ factors into the sliver state (D25-brane) and the $n$-th GMS soliton. Section 7 describes related results.

\section{The three string vertex in the presence of a constant background $B$ field}

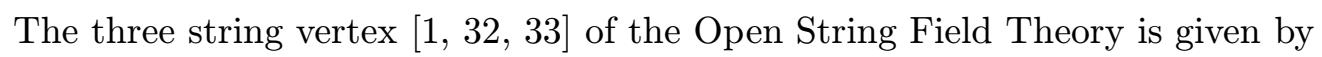

$$
\left|V_{3}\right\rangle=\int d^{26} p_{(1)} d^{26} p_{(2)} d^{26} p_{(3)} \delta^{26}\left(p_{(1)}+p_{(2)}+p_{(3)}\right) \exp (-E)|0, p\rangle_{123}
$$

where

$$
E=\sum_{r, s=1}^{3}\left(\frac{1}{2} \sum_{m, n \geq 1} \eta_{\mu \nu} a_{m}^{(r) \mu \dagger} V_{m n}^{r s} a_{n}^{(s) \nu \dagger}+\sum_{n \geq 1} \eta_{\mu \nu} p_{(r)}^{\mu} V_{0 n}^{r s} a_{n}^{(s) \nu \dagger}+\frac{1}{2} \eta_{\mu \nu} p_{(r)}^{\mu} V_{00}^{r s} p_{(s)}^{\nu}\right)
$$

Summation over the Lorentz indices $\mu, \nu=0, \ldots, 25$ is understood and $\eta$ denotes the flat Lorentz metric and the operators $a_{m}^{(r) \mu}, a_{m}^{(r) \mu \dagger}$ denote the non-zero modes matter oscillators of the $r$-th string, which satisfy

$$
\left[a_{m}^{(r) \mu}, a_{n}^{(s) \nu \dagger}\right]=\eta^{\mu \nu} \delta_{m n} \delta^{r s}, \quad m, n \geq 1
$$

$p_{(r)}$ is the momentum of the $r$-th string and $|0, p\rangle_{123} \equiv\left|p_{(1)}\right\rangle \otimes\left|p_{(2)}\right\rangle \otimes\left|p_{(3)}\right\rangle$ is the tensor product of the Fock vacuum states relative to the three strings. $\left|p_{(r)}\right\rangle$ is annihilated by the annihilation operators $a_{m}^{(r) \mu}$ and it is eigenstate of the momentum operator $\hat{p}_{(r)}^{\mu}$ with eigenvalue $p_{(r)}^{\mu}$. The normalization is

$$
\left\langle p_{(r)} \mid p_{(s)}^{\prime}\right\rangle=\delta_{r s} \delta^{26}\left(p+p^{\prime}\right)
$$

The coefficients $V_{M N}^{r s}(M(N)$ denotes from now on the couple $\{0, m\}(\{0, n\}))$ have been

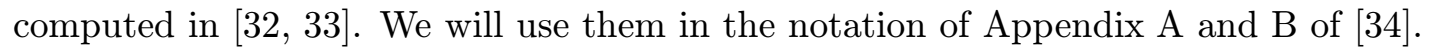

Our first goal is to find the new form of the coefficients $V_{M N}^{r s}$ when a constant $B$ field is switched on. We start from the simplest case, i.e. when $B$ is nonvanishing in two space directions, say the $24-$ th and 25 -th ones. Let us denote these directions with the Lorentz

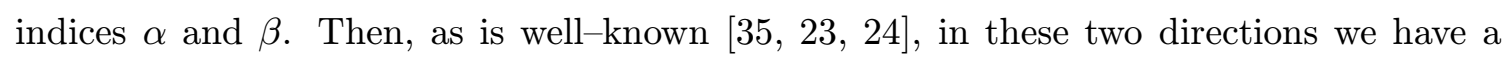
new effective metric $G_{\alpha \beta}$, the open string metric, as well as an effective antisymmetric parameter $\theta_{\alpha \beta}$, given by

$$
G^{\alpha \beta}=\left(\frac{1}{\eta+2 \pi \alpha^{\prime} B} \eta \frac{1}{\eta-2 \pi \alpha^{\prime} B}\right)^{\alpha \beta}, \quad \theta^{\alpha \beta}=-\left(2 \pi \alpha^{\prime}\right)^{2}\left(\frac{1}{\eta+2 \pi \alpha^{\prime} B} B \frac{1}{\eta-2 \pi \alpha^{\prime} B}\right)^{\alpha \beta}
$$


Henceforth we set $\alpha^{\prime}=1$, unless otherwise specified.

The three string vertex is modified only in the 24-th and 25-th direction, which, in view of the subsequent $\mathrm{D}$-brane interpretation, we call the transverse directions. We split the three string vertex into the tensor product of the perpendicular part and the parallel part

$$
\left|V_{3}\right\rangle=\left|V_{3, \perp}\right\rangle \otimes\left|V_{3, \|}\right\rangle
$$

The parallel part is the same as in the ordinary case and will not be re-discussed here. On the contrary we will describe in detail the perpendicular part of the vertex. We rewrite the exponent $E$ as $E=E_{\|}+E_{\perp}$, according to the above splitting. $E_{\perp}$ will be modified as follows

$$
\begin{aligned}
E_{\perp} \rightarrow E_{\perp}^{\prime} & =\sum_{r, s=1}^{3}\left(\frac{1}{2} \sum_{m, n \geq 1} G_{\alpha \beta} a_{m}^{(r) \alpha \dagger} V_{m n}^{r s} a_{n}^{(s) \beta \dagger}+\sum_{n \geq 1} G_{\alpha \beta} p_{(r)}^{\alpha} V_{0 n}^{r s} a_{n}^{(s) \beta \dagger}\right. \\
& \left.+\frac{1}{2} G_{\alpha \beta} p_{(r)}^{\alpha} V_{00}^{r s} p_{(s)}^{\beta}+\frac{i}{2} \sum_{r<s} p_{\alpha}^{(r)} \theta^{\alpha \beta} p_{\beta}^{(s)}\right)
\end{aligned}
$$

Next, as far as the zero modes are concerned, we pass from the momentum to the

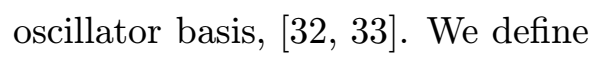

$$
a_{0}^{(r) \alpha}=\frac{1}{2} \sqrt{b} \hat{p}^{(r) \alpha}-i \frac{1}{\sqrt{b}} \hat{x}^{(r) \alpha}, \quad a_{0}^{(r) \alpha \dagger}=\frac{1}{2} \sqrt{b} \hat{p}^{(r) \alpha}+i \frac{1}{\sqrt{b}} \hat{x}^{(r) \alpha},
$$

where $\hat{p}^{(r) \alpha}, \hat{x}^{(r) \alpha}$ are the zero momentum and position operator of the $r$-th string, and we

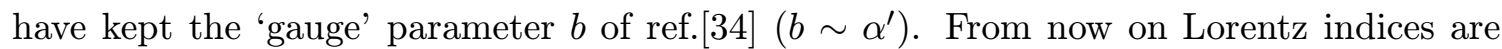
raised and lowered by means of the effective open string metric, for instance $p^{(r) \alpha}=G^{\alpha \beta} p_{\beta}^{(r)}$. We have

$$
\left[a_{M}^{(r) \alpha}, a_{N}^{(s) \beta \dagger}\right]=G^{\alpha \beta} \delta^{r s} \delta_{M N}, \quad N, M \geq 0
$$

Denoting by $\left|\Omega_{b, \theta}\right\rangle$ the oscillator vacuum $\left(a_{N}^{\alpha}\left|\Omega_{b, \theta}\right\rangle=0\right.$, for $N \geq 0$ ), the relation between the momentum basis and the oscillator basis is defined by

$$
\begin{aligned}
& \left|p^{24}\right\rangle_{123} \otimes\left|p^{25}\right\rangle_{123} \equiv\left|\left\{p^{\alpha}\right\}\right\rangle_{123}= \\
& \left(\frac{b}{2 \pi \sqrt{\operatorname{det} G}}\right)^{\frac{3}{2}} \exp \left[\sum_{r=1}^{3}\left(-\frac{b}{4} p_{\alpha}^{(r)} G^{\alpha \beta} p_{\beta}^{(r)}+\sqrt{b} a_{0}^{(r) \alpha \dagger} p_{\alpha}^{(r)}-\frac{1}{2} a_{0}^{(r) \alpha \dagger} G_{\alpha \beta} a_{0}^{(r) \beta \dagger}\right)\right]\left|\Omega_{b, \theta}\right\rangle
\end{aligned}
$$

Now we insert this equation inside $E_{\perp}^{\prime}$ and try to eliminate the momenta along the perpendicular directions by integrating them out. To this end we rewrite $E_{\perp}^{\prime}$ in the following way and, for simplicity, drop all the labels $\alpha, \beta$ and $r, s$ :

$E_{\perp}^{\prime}=\frac{1}{2} \sum_{m, n \geq 1} a_{m}^{\dagger} G V_{m n} a_{n}^{\dagger}+\sum_{n \geq 1} p V_{0 n} a_{n}^{\dagger}+\frac{1}{2} p\left[G^{-1}\left(V_{00}+\frac{b}{2}\right)+\frac{i}{2} \theta \epsilon \chi\right] p-\sqrt{b} p a_{0}^{\dagger}+\frac{1}{2} a_{0}^{\dagger} G a_{0}^{\dagger}$ 
where we have set $\theta^{\alpha \beta}=\epsilon^{\alpha \beta} \theta$ and introduced the matrices $\epsilon$ with entries $\epsilon^{\alpha \beta}$ (which represent the $2 \times 2$ antisymmetric symbol with $\epsilon_{2}^{1}=1$ ) and $\chi$ with entries

$$
\chi^{r s}=\left(\begin{array}{ccc}
0 & 1 & -1 \\
-1 & 0 & 1 \\
1 & -1 & 0
\end{array}\right)
$$

At this point we impose momentum conservation. There are three distinct ways to do that and eventually one has to (multiplicatively) symmetrize with respect to them. Let us start by setting $p_{3}=-p_{1}-p_{2}$ in $E_{\perp}^{\prime}$ and obtain an expression of the form

$$
p X_{00} p+\sum_{N \geq 0} p Y_{0 N} a_{N}^{\dagger}+\sum_{M, N \geq 0} a_{M}^{\dagger} Z_{M N} a_{N}^{\dagger}
$$

where, in particular, $X_{00}$ is given by

$$
X_{00}^{\alpha \beta, r s}=G^{\alpha \beta}\left(V_{00}+\frac{b}{2}\right) \eta^{r s}+i \frac{\theta}{4} \epsilon^{\alpha \beta} \epsilon^{r s}
$$

Here the indices $r, s$ take only the values 1,2 , and $\eta=\left(\begin{array}{cc}1 & 1 / 2 \\ 1 / 2 & 1\end{array}\right)$.

Now, as usual, we redefine $p$ so as eliminate the linear term in $\left(\overline{2} . \overline{2}_{1}\right)$. At this point we can easily perform the Gaussian integration over $p_{(1)}, p_{(2)}$, while the remnant of $\left(2 . \overline{2}_{1}\right)$ will be expressed in terms of the inverse of $X_{00}$ :

$$
\left(X_{00}^{-1}\right)^{\alpha \beta, r s}=\frac{2 A^{-1}}{4 a^{2}+3}\left(\frac{3}{2} G^{\alpha \beta}\left(\eta^{-1}\right)^{r s}-2 i a \epsilon^{\alpha \beta} \epsilon^{r s}\right)
$$

where

$$
A=V_{00}+\frac{b}{2}, \quad a=\frac{\theta}{4 A} .
$$

Let us use henceforth for the $B$ field the explicit form

$$
B_{\alpha \beta}=\left(\begin{array}{cc}
0 & B \\
-B & 0
\end{array}\right)
$$

so that

$$
\operatorname{DetG}=\left(1+(2 \pi B)^{2}\right)^{2}, \quad \theta=-(2 \pi)^{2} B, \quad a=-\frac{\pi^{2}}{A} B
$$

Now one has to symmetrize with respect to the three possibilities of imposing the momentum conservation. Remembering the factors due to integration over the momenta and collecting the results one gets for the three string vertex in the presence of a $B$ field

$$
\left|V_{3}\right\rangle^{\prime}=\left|V_{3, \perp}\right\rangle^{\prime} \otimes\left|V_{3, \|}\right\rangle
$$

$\left|V_{3, \|}\right\rangle$ is the same as in the ordinary case (without $B$ field), while

$$
\left|V_{3, \perp}\right\rangle^{\prime}=K_{2} e^{-E^{\prime}}|\tilde{0}\rangle
$$


with

$$
\begin{aligned}
K_{2} & =\frac{\sqrt{2 \pi b^{3}}}{A^{2}\left(4 a^{2}+3\right)}(\operatorname{Det} G)^{1 / 4}, \\
E^{\prime} & =\frac{1}{2} \sum_{r, s=1}^{3} \sum_{M, N \geq 0} a_{M}^{(r) \alpha \dagger} \nu_{\alpha \beta, M N}^{r s} a_{N}^{(s) \beta \dagger}
\end{aligned}
$$

and $|\tilde{0}\rangle=|0\rangle \otimes\left|\Omega_{b, \theta}\right\rangle$. The coefficients $\mathcal{V}_{M N}^{\alpha \beta, r s}$ are given by

$$
\begin{aligned}
& \mathcal{V}_{00}^{\alpha \beta, r s}=G^{\alpha \beta} \delta^{r s}-\frac{2 A^{-1} b}{4 a^{2}+3}\left(G^{\alpha \beta} \phi^{r s}-i a \epsilon^{\alpha \beta} \chi^{r s}\right) \\
& \mathcal{V}_{0 n}^{\alpha \beta, r s}=\frac{2 A^{-1} \sqrt{b}}{4 a^{2}+3} \sum_{t=1}^{3}\left(G^{\alpha \beta} \phi^{r t}-i a \epsilon^{\alpha \beta} \chi^{r t}\right) V_{0 n}^{t s} \\
& V_{m n}^{\alpha \beta, r s}=G^{\alpha \beta} V_{m n}^{r s}-\frac{2 A^{-1}}{4 a^{2}+3} \sum_{t, v=1}^{3} V_{m 0}^{r v}\left(G^{\alpha \beta} \phi^{v t}-i a \epsilon^{\alpha \beta} \chi^{v t}\right) V_{0 n}^{t s}
\end{aligned}
$$

where, by definition, $V_{0 n}^{r s}=V_{n 0}^{s r}$, and

$$
\phi=\left(\begin{array}{ccc}
1 & -1 / 2 & -1 / 2 \\
-1 / 2 & 1 & -1 / 2 \\
-1 / 2 & -1 / 2 & 1
\end{array}\right)
$$

while the matrix $\chi$ has been defined above (12.8'). These two matrices satisfy the algebra

$$
\chi^{2}=-2 \phi, \quad \phi \chi=\chi \phi=\frac{3}{2} \chi, \quad \phi^{2}=\frac{3}{2} \phi
$$

Next, let us notice that the above results can be easily extended to the case in which the transverse directions are more than two (i.e. the $24-$ th and 25 -th ones) and even. The canonical form of the transverse $B$ field is

$$
B_{\alpha \beta}=\left(\begin{array}{ccccc}
0 & B_{1} & & & \ldots \\
-B_{1} & 0 & & & \\
0 & & 0 & B_{2} & \\
\ldots & -B_{2} & 0 & \ldots \\
\ldots & & \ldots
\end{array}\right)
$$

It is not hard to see that each couple of conjugate transverse directions under this decomposition, can be treated in a completely independent way. The result is that each couple of directions $(26-i, 25-i)$, corresponding to the eigenvalue $B_{i}$, will be characterized by the same formulas $(2,19,2,20,2 . \overline{1} 1)$ above with $B$ replaced by $B_{i}$.

The properties of the new Neumann coefficients $\mathcal{V}_{N M}^{r s}$ are analyzed in Appendix A. Here we write down the results.

Let us introduce the twist matrix $C^{\prime}$ by $C_{M N}^{\prime}=(-1)^{M} \delta_{M N}$ and define

$$
X^{r s} \equiv C^{\prime} \mathcal{V}^{r s}, \quad r, s=1,2, \quad X^{11} \equiv X
$$

These matrices commute

$$
\left[X^{r s}, X^{r^{\prime} s^{\prime}}\right]=0
$$


and

$$
\left(X^{r s}\right)^{*}=\tilde{X}^{r s}, \text { i.e. } \quad\left(X^{r s}\right)^{\dagger}=X^{r s}
$$

Moreover we have the following properties, which mark a difference with the $B=0$ case,

$$
C^{\prime} \mathcal{V}^{r s}=\tilde{\mathcal{V}}^{s r} C^{\prime}, \quad C^{\prime} \mathcal{X}^{r s}=\tilde{X}^{s r} C^{\prime}
$$

where we recall that tilde denotes transposition with respect to the $\alpha, \beta$ indices. Finally one can prove that

$$
\begin{aligned}
& X^{11}+X^{12}+X^{21}=\mathbb{I} \\
& X^{12} X^{21}=\left(X^{11}\right)^{2}-X \\
& \left(X^{12}\right)^{2}+\left(X^{21}\right)^{2}=\mathbb{I}-\left(X^{11}\right)^{2} \\
& \left(X^{12}\right)^{3}+\left(X^{21}\right)^{3}=2\left(X^{11}\right)^{3}-3\left(X^{11}\right)^{2}+\mathbb{I}
\end{aligned}
$$

In the matrix products of these identities, as well as throughout the paper, the indices $\alpha, \beta$

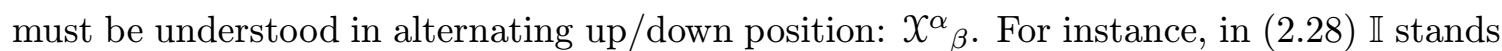
for $\delta^{\alpha}{ }_{\beta} \delta_{M N}$.

\section{The squeezed state solution}

In this section we wish to find a solution to the equation of motion $|\Psi\rangle *|\Psi\rangle=|\Psi\rangle$ in the

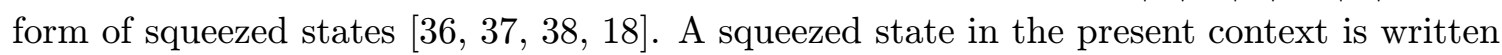
as

$$
|S\rangle=\left|S_{\perp}\right\rangle \otimes\left|S_{\|}\right\rangle
$$

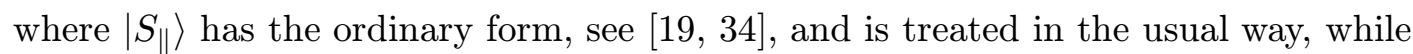

$$
\left|S_{\perp}\right\rangle=\mathcal{N}^{2} \exp \left(-\frac{1}{2} \sum_{M, N \geq 0} a_{M}^{\alpha \dagger} \mathcal{S}_{\alpha \beta, M N} a_{N}^{\beta \dagger}\right)\left|\Omega_{b, \theta}\right\rangle
$$

The $*$ product of two such states, labeled ${ }_{1}$ and ${ }_{2}$, is

$$
\left|S_{\perp}^{\prime}\right\rangle=\left|S_{1, \perp}\right\rangle *\left|S_{2, \perp}\right\rangle=\frac{K_{2}\left(\mathcal{N}_{1} \mathcal{N}_{2}\right)^{2}}{\operatorname{DET}(\mathbf{I}-\Sigma \mathcal{V})^{1 / 2}} \exp \left(-\frac{1}{2} \sum_{M, N \geq 0} a_{M}^{\alpha \dagger} \mathcal{S}_{\alpha \beta, M N}^{\prime} a_{N}^{\beta \dagger}\right)|\tilde{0}\rangle
$$

where, in matrix notation which includes both the indices $N, M$ and $\alpha, \beta$,

$$
\mathcal{S}^{\prime}=\mathcal{V}^{11}+\left(\mathcal{V}^{12}, \mathcal{V}^{21}\right)(\mathbf{I}-\Sigma \mathcal{V})^{-1} \Sigma\left(\begin{array}{l}
\mathcal{V}^{21} \\
\mathcal{V}^{12}
\end{array}\right)
$$

In RHS of these equations

$$
\Sigma=\left(\begin{array}{cc}
C^{\prime} \mathcal{S}_{1} C^{\prime} & 0 \\
0 & C^{\prime} \mathcal{S}_{2} C^{\prime}
\end{array}\right), \quad \mathcal{V}=\left(\begin{array}{cc}
\mathcal{V}^{11} & \mathcal{V}^{12} \\
\mathcal{V}^{21} & \mathcal{V}^{22}
\end{array}\right)
$$


and $\mathbf{I}_{\beta, M N}^{\alpha, r s}=\delta_{\beta}^{\alpha} \delta_{M N} \delta^{r s}, r, s=1,2$. DET is the determinant with respect to all indices. To reach the form (3.4) one has to use cyclicity of $\mathcal{V}^{r s}$ (property (ii) in Appendix A).

Let us now discuss the squeezed state solution of the equation $|\Psi\rangle *|\Psi\rangle=|\Psi\rangle$ in the matter sector. In order for this to be satisfied with the above states $|S\rangle$, we must first impose

$$
\mathcal{S}_{1}=\mathcal{S}_{2}=\mathcal{S}^{\prime} \equiv \mathcal{S}
$$

and then suitably normalize the resulting state. Then $(\underline{3} . \overline{1})$ becomes an equation for $\mathcal{S}$, i.e.

$$
\tilde{\mathcal{S}}=\mathcal{V}^{11}+\left(\mathcal{V}^{12}, \mathcal{V}^{21}\right)(\mathbf{I}-\Sigma \mathcal{V})^{-1} \Sigma\left(\begin{array}{l}
\mathcal{V}^{21} \\
\mathcal{V}^{12}
\end{array}\right)
$$

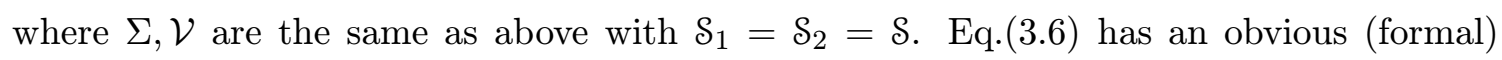
solution by iteration. However in ref. [i] $\left.\underline{1}_{1}^{-} \bar{i}\right]$ it was shown that it is possible to obtain the solution in compact form by 'abelianizing' the problem. Notwithstanding the differences

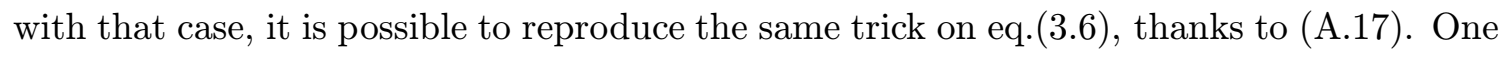
denotes $C^{\prime} \mathcal{V}^{r s}$ by $X^{r s}$ and $C^{\prime} \mathcal{S}$ by $\mathcal{T}$, and assumes that $\left[X^{r s}, \mathcal{T}\right]=0$ (of course this has to be checked a posteriori). Notice however that we cannot assume that $C^{\prime}$ commutes with $\mathcal{S}$, but we assume that $C^{\prime} \mathcal{S}=\tilde{\mathcal{S}} C^{\prime}$. By multiplying $\left(\bar{\beta}_{0} . \overline{6}_{i}^{\prime}\right)$ from the left by $C^{\prime}$ we get:

$$
\mathcal{T}=X^{11}+\left(X^{12}, X^{21}\right)(\mathbf{I}-\Sigma \mathcal{V})^{-1}\left(\begin{array}{l}
\mathcal{T} X^{21} \\
\mathcal{T} X^{12}
\end{array}\right)
$$

For instance $\tilde{\mathcal{S}} \mathcal{V}^{12}=\tilde{S} C^{\prime} C^{\prime} \mathcal{V}^{12}=\mathfrak{T} \mathcal{X}^{12}$, etc. In the same way,

$$
(\mathbf{I}-\Sigma \mathcal{V})^{-1}=\left(\begin{array}{cc}
\mathbb{I}-\mathcal{T} \mathcal{X}^{11} & -\mathcal{T} \mathcal{X}^{12} \\
-\mathcal{T} \mathcal{X}^{21} & \mathbb{I}-\mathcal{T} \mathcal{X}^{11}
\end{array}\right)^{-1}
$$

where $\mathbb{I}_{\beta, M N}^{\alpha}=\delta_{\beta}^{\alpha} \delta_{M N}$. Now all the entries are commuting matrices, so the inverse can be calculated straight away.

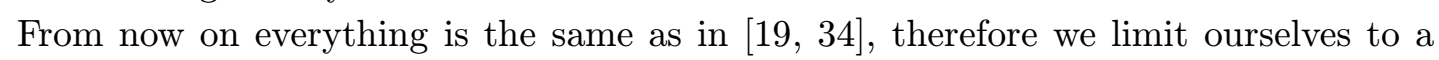
quick exposition. One arrives at an equation only in terms of $\mathcal{T}$ and $X \equiv \mathcal{X}^{11}$ :

$$
(\mathcal{T}-\mathbb{I})\left(X \mathcal{T}^{2}-(\mathbb{I}+X) \mathcal{T}+X\right)=0
$$

This gives two solutions:

$$
\begin{aligned}
\mathcal{T} & =\mathbb{I} \\
\mathcal{T} & =\frac{1}{2 X}(\mathbb{I}+X-\sqrt{(\mathbb{I}+3 X)(\mathbb{I}-X)})
\end{aligned}
$$

The third solution, with a + sign in front of the square root, is not acceptable, as explained

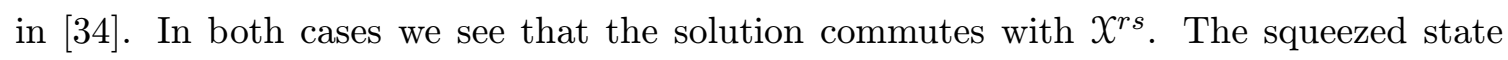
solution we are looking for is, in both cases, $\mathcal{S}=C^{\prime} \mathcal{T}$. As for $\left(3_{-1}^{3} . \overline{9}\right)$, it is easy to see that it leads to the identity state. Therefore, from now on we will consider (3.10i) alone.

Now, let us deal with the normalization of $\left|S_{\perp}\right\rangle$. Imposing $\left|S_{\perp}\right\rangle *\left|S_{\perp}\right\rangle=\left|S_{\perp}\right\rangle$ we find

$$
\mathcal{N}^{2}=K_{2}^{-1} \operatorname{DET}(\mathbf{I}-\Sigma \mathcal{V})^{1 / 2}
$$


Replacing the solution in it one finds

$$
\operatorname{DET}(\mathbf{I}-\Sigma \mathcal{V})=\operatorname{Det}((\mathbb{I}-\mathcal{X})(\mathbb{I}+\mathcal{T}))
$$

Det denotes the determinant with respect to the indices $\alpha, \beta, M, N$. Using this equation and $(\overline{2} \overline{1} \overline{1} \overline{1})$, and borrowing from $\left[\overline{3} \overline{4}_{i}^{i}\right]$ the expression for $\left|S_{\|}\right\rangle$, one finally gets for the 23 dimensional tachyonic lump, which we denote by

$$
\begin{aligned}
|S\rangle= & \left\{\operatorname{det}(1-X)^{1 / 2} \operatorname{det}(1+T)^{1 / 2}\right\}^{24} \exp \left(-\frac{1}{2} \eta_{\bar{\mu} \bar{\nu}} \sum_{m, n \geq 1} a_{m}^{\bar{\mu} \dagger} S_{m n} a_{n}^{\bar{\nu} \dagger}\right)|0\rangle \otimes \\
& \frac{A^{2}\left(3+4 a^{2}\right)}{\sqrt{2 \pi b^{3}}(\operatorname{Det} G)^{1 / 4}}\left(\operatorname{Det}(\mathbb{I}-X)^{1 / 2} \operatorname{Det}(\mathbb{I}+\mathcal{T})^{1 / 2}\right) \exp \left(-\frac{1}{2} \sum_{M, N \geq 0} a_{M}^{\alpha \dagger} \mathcal{S}_{\alpha \beta, M N} a_{N}^{\beta \dagger}\right)|\tilde{0}\rangle,
\end{aligned}
$$

where $\mathcal{S}=C^{\prime} \mathcal{T}$ and $\mathcal{T}$ is given by $\left(3^{3} \cdot 1\right)$. The quantities in the first line are defined in ref.[ $\overline{3} \overline{3} \overline{4}]$ with $\bar{\mu}, \bar{\nu}=0, \ldots 23$ denoting the parallel directions to the lump.

The value of the action corresponding to (3.12) is easily calculated

$$
\begin{aligned}
\mathcal{S}_{\mathcal{S}}= & \mathcal{K} \frac{V^{(24)}}{(2 \pi)^{24}}\left\{\operatorname{det}(1-X)^{3 / 4} \operatorname{det}(1+3 X)^{1 / 4}\right\}^{24} \\
& \cdot \frac{A^{4}\left(3+4 a^{2}\right)^{2}}{2 \pi b^{3}(\operatorname{Det} G)^{1 / 2}} \operatorname{Det}(\mathbb{I}-X)^{3 / 4} \operatorname{Det}(\mathbb{I}+3 X)^{1 / 4}
\end{aligned}
$$

where $V^{(24)}$ is the volume along the parallel directions and $\mathcal{K}$ is the constant of eq.(1).

Finally, let $\mathfrak{e}$ denote the energy per unit volume, which coincides with the brane tension when $B=0$. Then one can compute the ratio of the D23-brane energy density $\mathfrak{e}_{23}$ to the D25-brane energy density $\mathfrak{e}_{25}$;

$$
\begin{aligned}
\frac{\mathfrak{e}_{23}}{\mathfrak{e}_{25}} & =\frac{(2 \pi)^{2}}{(\operatorname{Det} G)^{1 / 4}} \cdot \mathcal{R} \\
\mathcal{R} & =\frac{A^{4}\left(3+4 a^{2}\right)^{2}}{2 \pi b^{3}(\operatorname{Det} G)^{1 / 4}} \frac{\operatorname{Det}(\mathbb{I}-X)^{3 / 4} \operatorname{Det}(\mathbb{I}+3 X)^{1 / 4}}{\operatorname{det}(1-X)^{3 / 2} \operatorname{det}(1+3 X)^{1 / 2}}
\end{aligned}
$$

If the quantity $\mathcal{R}$ equals 1 , this equation is exactly what is expected for the ratio of a flat static D25-brane action and a D23-brane action per unit volume in the presence of

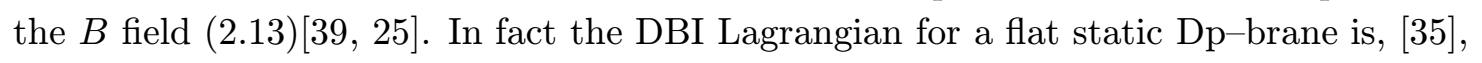

$$
\mathcal{L}_{D B I}=\frac{1}{g_{s}(2 \pi)^{p}} \sqrt{\operatorname{Det}(1+2 \pi B)}
$$

where $g_{s}$ is the closed string coupling. Substituting (2.13) and taking the ratio the claim

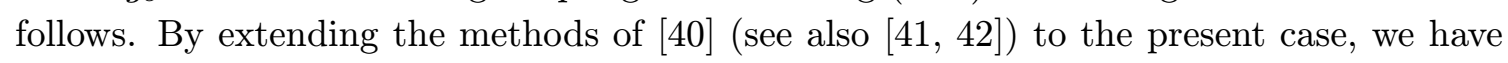

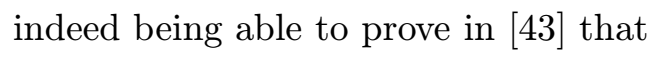

$$
\mathcal{R}=1
$$

thus adding evidence to the interpretation of $|S\rangle$, given by (3.12), as a D23-brane in the presence of a background $B$ field. A further confirmation of this interpretation could 
be obtained from the study of the spectrum of modes leaving on the brane, which can

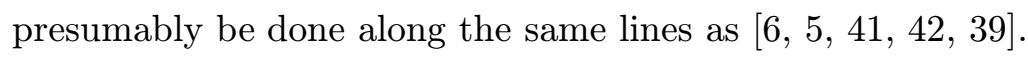

To end this section let us briefly discuss the generalization of the above results to lower dimensional lumps. As remarked at the end of section 2, every couple of transverse directions corresponding to an eigenvalue $B_{i}$ of the field $B$ can be treated in the same way as the 24-th and 25-th directions. One has simply to replace in the above formulas $B$ with $B_{i}$. The derivation of the above formulas for the case of $25-2 i$ dimensional lumps is straightforward.

\section{Some effects of the $B$ field}

In this section we would like to show that what we have obtained so far is not merely a formal replica of the same calculation without $B$ field, but that it significantly affects the lumps solutions. Precisely we would like to show that a $B$ field has the effect of smoothing out some of the singularities that appear in the VSFT, in particular in the low energy limit.

In [4] 4 in was shown that the geometry of the lower-dimensional lump states representing Dp-branes is singular. This can be seen both in the zero slope limit $\alpha^{\prime} \rightarrow 0$ and as an exact result. It can be briefly stated by saying that the midpoint of the string is confined on the hyperplane of vanishing transverse coordinates. It is therefore interesting to see whether the presence of a $B$ field modifies this situation. Moreover, as explained in the introduction, soliton solutions of field theories defined on a noncommutative space describe Dp-branes $([\underline{4} \overline{4} \overline{5}],[2 \overline{2} \overline{0}])$. It is then interesting to see if we can recover the simplest

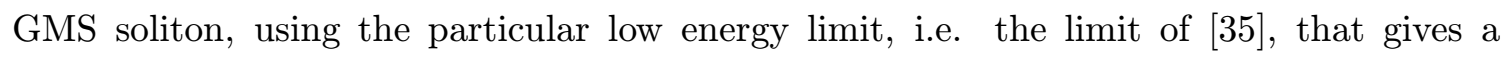
noncommutative field theory from a string theory with a $B$ field turned on.

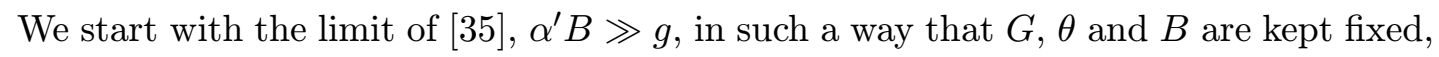
which we represent by means of a parameter $\epsilon$ going to 0 as in [44in] $\left(\alpha^{\prime} \sim \epsilon^{2}\right)$. We write the closed string metric $g_{\alpha \beta}$ as $g \delta_{\alpha \beta}$. We could also choose to parametrize the $\alpha^{\prime} B \gg g$ condition by sending $B$ to infinity, keeping $g$ and $\alpha^{\prime}$ fixed and operating a rescaling of the

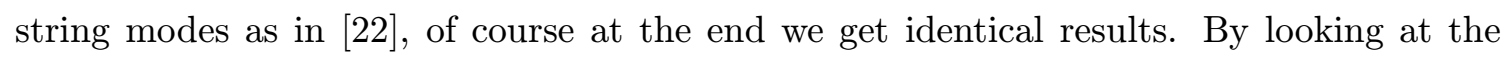
exponential of the 3 -string field theory vertex in the presence of a $B$ field

$$
\begin{aligned}
& \sum_{r, s=1}^{3}\left(\frac{1}{2} \sum_{m, n \geq 1} G_{\alpha \beta} a_{m}^{(r) \alpha \dagger} V_{m n}^{r s} a_{n}^{(s) \beta \dagger}+\sqrt{\alpha^{\prime}} \sum_{n \geq 1} G_{\alpha \beta} p_{(r)}^{\alpha} V_{0 n}^{r s} a_{n}^{(s) \beta \dagger}\right. \\
& \left.\quad+\alpha^{\prime} \frac{1}{2} G_{\alpha \beta} p_{(r)}^{\alpha} V_{00}^{r s} p_{(s)}^{\beta}+\frac{i}{2} \sum_{r<s} p_{\alpha}^{(r)} \theta^{\alpha \beta} p_{\beta}^{(s)}\right)
\end{aligned}
$$

we see that the limit is characterized by the rescalings

$$
\begin{aligned}
& V_{m n} \rightarrow V_{m n} \\
& V_{m 0} \rightarrow \epsilon V_{m 0} \\
& V_{00} \rightarrow \epsilon^{2} V_{00}
\end{aligned}
$$


The dependence of $G_{\alpha \beta}$ and $\theta^{\alpha \beta}$ on $g, \alpha^{\prime}$ and $B$ is understood. We will make it explicit at the end of our calculations in the form

$$
G_{\alpha \beta}=\frac{\left(2 \pi \alpha^{\prime} B\right)^{2}}{g} \delta_{\alpha \beta}, \quad \theta=\frac{1}{B}
$$

Substituting the leading behaviors of $V_{M N}$ in eqs.( $(2.21)$, and keeping in mind that $A=$ $V_{00}+\frac{b}{2}$, the coefficients $\mathcal{V}_{M N}^{\alpha \beta, r s}$ become

$$
\begin{aligned}
& V_{00}^{\alpha \beta, r s} \rightarrow G^{\alpha \beta} \delta^{r s}-\frac{4}{4 a^{2}+3}\left(G^{\alpha \beta} \phi^{r s}-i a \epsilon^{\alpha \beta} \chi^{r s}\right) \\
& \mathcal{V}_{0 n}^{\alpha \beta, r s} \rightarrow 0 \\
& \mathcal{V}_{m n}^{\alpha \beta, r s} \rightarrow G^{\alpha \beta} V_{m n}^{r s}
\end{aligned}
$$

We see that the squeezed state (13.12i) factorizes in two parts: the coefficients $\nu_{m n}^{\alpha \beta, 11}$ reconstruct the full 25 dimensional sliver, while the coefficients $\mathcal{V}_{00}^{\alpha \beta, 11}$ take a very simple form

$$
\mathcal{S}_{00}^{\alpha \beta}=\frac{2|a|-1}{2|a|+1} G^{\alpha \beta} \equiv s G^{\alpha \beta}
$$

In the $\epsilon \rightarrow 0$ limit we also have

$$
\operatorname{Det}(\mathbb{I}-X)^{1 / 2} \operatorname{Det}(\mathbb{I}+\mathcal{T})^{1 / 2} \rightarrow \frac{4}{4 a^{2}+3} \operatorname{det}(1-X) \frac{4 a}{2 a+1} \operatorname{det}(1+T)
$$

The complete lump state in this limit will be denoted by $|\hat{\mathcal{S}}\rangle$, and as a consequence of eq.(3.12) and these equations, it will take the form

$$
\begin{aligned}
|\hat{\mathcal{S}}\rangle= & \left\{\operatorname{Det}(1-X)^{1 / 2} \operatorname{Det}(1+T)^{1 / 2}\right\}^{26} \exp \left(-\frac{1}{2} G_{\mu \nu} \sum_{m, n \geq 1} a_{m}^{\mu \dagger} S_{m n} a_{n}^{\nu \dagger}\right)|0\rangle \otimes \\
& \frac{4 a}{2 a+1} \frac{b^{2}}{\sqrt{2 \pi b^{3}}(\operatorname{Det} G)^{1 / 4}} \exp \left(-\frac{1}{2} s a_{0}^{\alpha \dagger} G_{\alpha \beta} a_{0}^{\beta \dagger}\right)\left|\Omega_{b, \theta}\right\rangle,
\end{aligned}
$$

where $\mu, \nu=0, \ldots 25$ and $G_{\mu \nu}=\eta_{\bar{\mu} \bar{\nu}} \otimes G_{\alpha \beta}$. The first line of the RHS of this equation is nothing but the sliver state $|\Xi\rangle$, which represents the D25-brane. The norm of the lump is now regularized by the presence of $a$ which is directly proportional to $B: a=-\frac{\pi^{2}}{A} B$. Using

$$
|x\rangle=\sqrt{\frac{2 \sqrt{\operatorname{Det} G}}{b \pi}} \exp \left[-\frac{1}{b} x^{\alpha} G_{\alpha \beta} x^{\beta}-\frac{2}{\sqrt{b}} i a_{0}^{\alpha \dagger} G_{\alpha \beta} x^{\beta}+\frac{1}{2} a_{0}^{\alpha \dagger} G_{\alpha \beta} a_{0}^{\beta \dagger}\right]\left|\Omega_{b, \theta}\right\rangle
$$

we can calculate the projection onto the basis of position eigenstates of the transverse part of the lump state

$$
\begin{aligned}
\left\langle x\left|e^{-\frac{s}{2}\left(a_{0}^{\dagger}\right)^{2}}\right| \Omega_{b, \theta}\right\rangle & =\sqrt{\frac{2 \sqrt{\operatorname{Det} G}}{b \pi}} \frac{1}{1+s} e^{-\frac{1-s}{1+s} \frac{1}{b} x^{\alpha} x^{\beta} G_{\alpha \beta}} \\
& =\sqrt{\frac{2 \sqrt{\operatorname{Det} G}}{b \pi}} \frac{1}{1+s} e^{-\frac{1}{2|a| b} x^{\alpha} x^{\beta} G_{\alpha \beta}}
\end{aligned}
$$


The transverse part of the lump state in the $x$ representation is then

$$
\left\langle x \mid \hat{\mathcal{S}}_{\perp}\right\rangle=\frac{1}{\pi} e^{-\frac{1}{2|a| b} x^{\alpha} x^{\beta} G_{\alpha \beta}}\left|\Xi_{\perp}\right\rangle
$$

Using now the form (14.3i) of $G_{\alpha \beta}$ and $\theta^{\alpha \beta}$ and the explicit expression for $a$ in terms of $g$ and $\alpha^{\prime},\left[{ }^{4} \overline{6} \bar{i}\right]$

$$
a=\frac{\theta}{4 A} \sqrt{\operatorname{Det} G}=-\frac{2 \pi^{2}\left(\alpha^{\prime}\right)^{2} B}{b g}
$$

we obtain the simplest soliton solution of [4든

$$
e^{-\frac{1}{2|a| b} x^{\alpha} x^{\beta} G_{\alpha \beta}} \rightarrow e^{-\frac{x^{\alpha} x^{\beta} \delta_{\alpha \beta}}{|\theta|}}
$$

which corresponds to the $|0\rangle\langle 0|$ projector in the harmonic oscillator Hilbert space (see Introduction), and is a projector on a space endowed with a Moyal product.

In this way the $B$ field provides a regularization of (4.12), as compared to [4] beneficial effect of the $B$ field is confirmed by the fact that the projector ( annihilated by $x_{0}$

$$
\begin{aligned}
x_{0} \exp \left(-\frac{1}{2} s a_{0}^{\alpha \dagger} G_{\alpha \beta} a_{0}^{\beta \dagger}\right)\left|\Omega_{b, \theta}\right\rangle & =i \frac{\sqrt{b}}{2}\left(a_{0}-a_{0}^{\dagger}\right) \exp \left(-\frac{1}{2} s a_{0}^{\alpha \dagger} G_{\alpha \beta} a_{0}^{\beta \dagger}\right)\left|\Omega_{b, \theta}\right\rangle \\
& =-i \frac{\sqrt{b}}{2}\left[\frac{4 a}{2 a+1}\right] a_{0}^{\dagger} \exp \left(-\frac{1}{2} s a_{0}^{\alpha \dagger} G_{\alpha \beta} a_{0}^{\beta \dagger}\right)\left|\Omega_{b, \theta}\right\rangle
\end{aligned}
$$

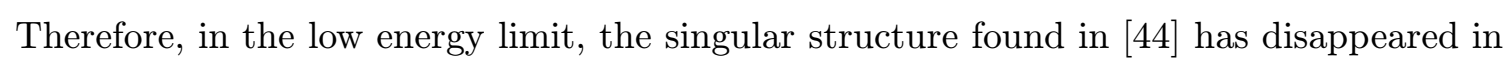
the presence of a nonvanishing $B$ field. This is actually not true only in the low energy limit, but is an exact result, as was shown in [ī $\underline{4} \overline{3}]$.

\section{More lumps in VSFT}

In the two previous sections we have constructed a 23-dimensional lump solution, which we have interpreted as a D23-brane. In the low energy limit this solution in the coordinate basis, turned out to be the simplest (two-dimensional) GMS soliton multiplied by a translational invariant solution which represents the D25-brane. The question we want to deal with here is whether there are other lump solutions that correspond to the higher order GMS solitons. The answer is affirmative. We will construct an infinite sequence of them, denoted $\left|\Lambda_{n}\right\rangle$. These new star algebra projectors are D23-branes, constructed out of (3.12) and parallel to it. In the low energy limit they give rise to the full series of GMS solitons. We will construct them and prove that they satisfy the remarkable identities (11.11.12i).

In order to construct these new solutions we need a new ingredient, given by the Fock space projectors similar to those introduced in [i] ${ }_{1}^{1}$ in. We define them only along the transverse directions

$$
\begin{aligned}
& \rho_{1}=\frac{1}{(\mathbb{I}+\mathcal{T})(\mathbb{I}-X)}\left[X^{12}(\mathbb{I}-\mathcal{T} X)+\mathcal{T}\left(X^{21}\right)^{2}\right] \\
& \rho_{2}=\frac{1}{(\mathbb{I}+\mathcal{T})(\mathbb{I}-X)}\left[X^{21}(\mathbb{I}-\mathcal{T} X)+\mathcal{T}\left(X^{12}\right)^{2}\right]
\end{aligned}
$$


They satisfy

$$
\rho_{1}^{2}=\rho_{1}, \quad \rho_{2}^{2}=\rho_{2}, \quad \rho_{1}+\rho_{2}=\mathbb{I}
$$

i.e. they project onto orthogonal subspaces. Moreover, if we use the superscript ${ }^{T}$ to denote transposition with respect to the indices $N, M$ and $\alpha, \beta$, we have

$$
\rho_{1}^{T}=\tilde{\rho}_{1}=C^{\prime} \rho_{2} C^{\prime}, \quad \rho_{2}^{T}=\tilde{\rho}_{2}=C^{\prime} \rho_{1} C^{\prime} .
$$

and

$$
\begin{aligned}
& \rho_{i}^{\dagger}=\rho_{i}, \quad \text { i.e. } \quad \rho_{i}^{*}=\tilde{\rho}_{i}, \quad i=1,2 \\
& \tau \rho_{i}=\tilde{\rho}_{i} \tau, \quad i=1,2
\end{aligned}
$$

where $^{*}$ denote complex conjugation and ${ }^{\dagger}={ }^{* T}$. Moreover $\tau$ is the matrix $\tau=\left\{\tau_{\alpha}{ }^{\beta}\right\}=$ $\left(\begin{array}{cc}1 & 0 \\ 0 & -1\end{array}\right)$. We recall that in the absence of the $B$ field, it has been shown that $\rho_{1}, \rho_{2}$

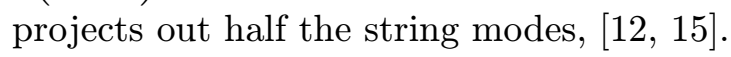

With all these ingredients we can now move on, give a precise definition of the $\left|\Lambda_{n}\right\rangle$ states and demonstrate the properties announced above.

To define the states $\left|\Lambda_{n}\right\rangle$ we start from the lump solution (3.12i). I.e. we take $\left|\Lambda_{0}\right\rangle=$ $|\mathcal{S}\rangle$. However, in the following, we will limit ourselves only to the transverse part of it, the parallel one being universal and irrelevant for our construction. We will denote the transverse part by $\left|\mathcal{S}_{\perp}\right\rangle$.

First we introduce two 'vectors' $\xi=\left\{\xi_{N \alpha}\right\}$ and $\zeta=\left\{\zeta_{N \alpha}\right\}$, which are chosen to satisfy the conditions

$$
\rho_{1} \xi=0, \quad \rho_{2} \xi=\xi, \quad \text { and } \quad \rho_{1} \zeta=0, \quad \rho_{2} \zeta=\zeta,
$$

Next we define

$$
\mathbf{x}=\left(a^{\dagger} \tau \xi\right)\left(a^{\dagger} C^{\prime} \zeta\right)=\left(a_{N}^{\alpha \dagger} \tau_{\alpha}^{\beta} \xi_{N \beta}\right)\left(a_{N}^{\alpha \dagger} C_{N M}^{\prime} \zeta_{M \alpha}\right)
$$

and introduce the Laguerre polynomials $L_{n}(z)$, of the generic variable $z$. The definition of $\left|\Lambda_{n}\right\rangle$ is as follows

$$
\left|\Lambda_{n}\right\rangle=(-\kappa)^{n} L_{n}\left(\frac{\mathbf{x}}{\kappa}\right)\left|\mathcal{S}_{\perp}\right\rangle
$$

As part of the definition of $\left|\Lambda_{n}\right\rangle$ we require the two following conditions to be satisfied

$$
\xi^{T} \tau \frac{1}{\mathbb{I}-\mathcal{T}^{2}} \zeta=-1, \quad \xi^{T} \tau \frac{\mathcal{T}}{\mathbb{I}-\mathcal{T}^{2}} \zeta=-\kappa
$$

Hermiticity for $\left|\Lambda_{n}\right\rangle$ requires that

$$
\left(a \tau \xi^{*}\right)\left(a C^{\prime} \zeta^{*}\right)=\left(a \tau C^{\prime} \xi\right)(a \zeta)
$$

This condition admits the solution

$$
\zeta=\tau \xi^{*}
$$


which we will assume throughout the rest of the paper, even though it will be left implicit for notational simplicity. Eq. (

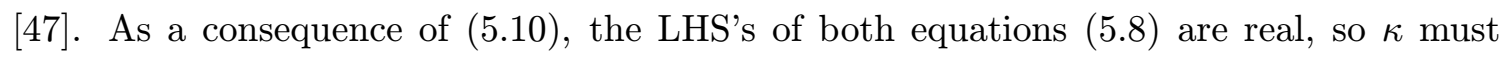
be real too. Let us show this for instance for the first equation, since for the second no significant modification is needed:

$$
\left(\xi^{T} \tau \frac{1}{\mathbb{I}-\mathcal{T}^{2}} \zeta\right)^{*}=\xi^{T *} \tau \frac{1}{\mathbb{I}-\left(\mathcal{T}^{*}\right)^{2}} \zeta^{*}=\zeta^{T} \frac{1}{\mathbb{I}-\left(\mathcal{T}^{*}\right)^{2}} \tau \xi=\xi^{T} \tau \frac{1}{\mathbb{I}-\left(\mathcal{T}^{\dagger}\right)^{2}} \zeta=\xi^{T} \tau \frac{1}{\mathbb{I}-\mathcal{T}^{2}} \zeta
$$

where the second equality is obtained by replacement of $(5.10)$, and the third by transposition.

In Appendix B we give the rather technical proof that (1.11) is satisfied. As for the

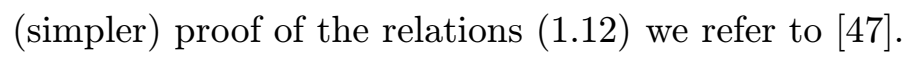

Before we pass to the low energy limit, let us make a comment on the definition of $\left|\Lambda_{n}\right\rangle$, wherein a central role is played by the Laguerre polynomials. While the true rationale of this role eludes us, it is possible to prove that the form of the definition $\left|\Lambda_{n}\right\rangle$ (together with

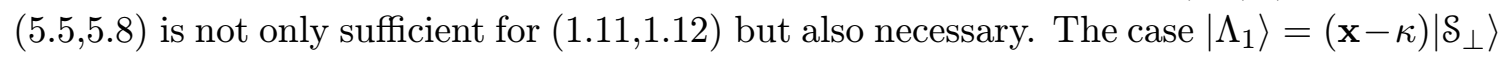
was discussed in [12int. The next most complicated state is

$$
\left(\alpha+\beta \mathbf{x}+\gamma \mathbf{x}^{2}\right)\left|\mathcal{S}_{\perp}\right\rangle
$$

The conditions this state has to satisfy in order to define a $\left|\Lambda_{2}\right\rangle$ that obeys (1.11,1,12i) for

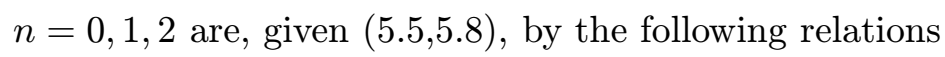

$$
-2(\alpha)^{1 / 2}=\beta, \quad \gamma=\frac{1}{2}
$$

Then, putting $\alpha=\kappa$

$$
\left|\mathcal{P}^{\prime}\right\rangle=\left(\kappa^{2}-2 \kappa \mathbf{x}+\frac{1}{2} \mathbf{x}^{2}\right)\left|\mathcal{S}_{\perp}\right\rangle
$$

The polynomial in the RHS is nothing but the second Laguerre polynomial of $\mathbf{x} / \kappa$ multiplied by $\kappa^{2}$. In fact using Mathematica it is easy to extend this analysis for $n$ as large as one wishes.

Finally let us remark that the relations demonstrated in this section, in particular $(1,11,1.121)$, are true for any value of $B$, therefore also for $B=0$.

\section{The GMS solitons}

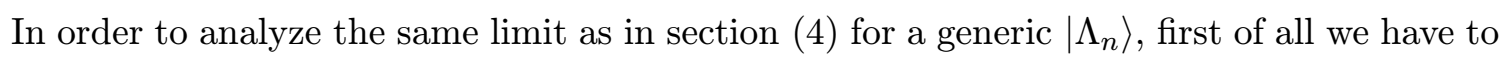
find the low energy limit of the projectors $\rho_{1}, \rho_{2}$. In this limit these two projectors factorize into the zero mode and non-zero mode part. The former is given by

$$
\left(\rho_{1}\right)_{00}^{\alpha \beta} \rightarrow \frac{1}{2}\left[G^{\alpha \beta}+i \epsilon^{\alpha \beta}\right], \quad\left(\rho_{2}\right)_{00}^{\alpha \beta} \rightarrow \frac{1}{2}\left[G^{\alpha \beta}-i \epsilon^{\alpha \beta}\right],
$$

Now, we take, in the definition $\left(\overline{5} . \bar{\sigma}^{\hat{j}}\right), \xi=\hat{\xi}+\bar{\xi}$ and $\zeta=\hat{\zeta}+\bar{\zeta}$, where $\bar{\xi}, \bar{\zeta}$ are such that they vanish in the limit $\alpha^{\prime} \rightarrow 0$. Then we make the choice $\hat{\xi}_{n}=\hat{\zeta}_{n}=0, \forall n>0$ 


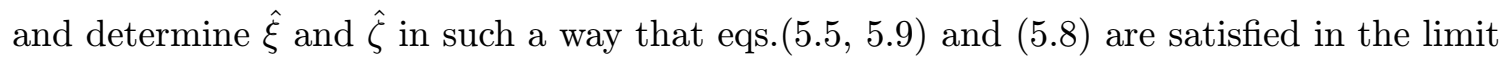
$\alpha^{\prime} \rightarrow 0$. We are assuming here that there exist solutions of the problem at $\alpha^{\prime} \neq 0$ that take precisely this specific form when $\alpha^{\prime} \rightarrow 0$. This is a plausible assumption since the $\alpha^{\prime}$ dependence is smooth in all the involved quantities. In any case it is not hard to construct examples of this fact: for instance $\xi=\rho_{2} \hat{\xi}$ satisfies the above requirements to order zero

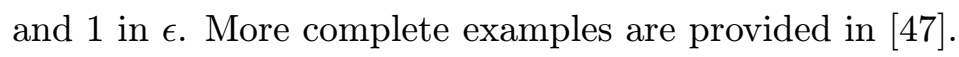

Now, in the field theory limit the conditions (1.5.5. become

$$
\hat{\xi}_{0,24}+i \hat{\xi}_{0,25}=0, \quad \hat{\zeta}_{0,24}+i \hat{\zeta}_{0,25}=0
$$

From now on we set $\hat{\xi}_{0}=\hat{\xi}_{0,25}=-i \hat{\xi}_{0,24}$ and, similarly, $\hat{\zeta}_{0}=\hat{\zeta}_{0,25}=-i \hat{\zeta}_{0,24}$. The conditions (1) -

$$
\begin{aligned}
& \xi^{T} \tau \frac{1}{\mathbb{I}-\mathcal{T}^{2}} \zeta \rightarrow-\frac{1}{1-s^{2}} \frac{2}{\sqrt{\operatorname{Det} G}} \hat{\xi}_{0} \hat{\zeta}_{0}=-1 \\
& \xi^{T} \tau \frac{\mathcal{T}}{\mathbb{I}-\mathcal{T}^{2}} \zeta \rightarrow-\frac{s}{1-s^{2}} \frac{2}{\sqrt{\operatorname{Det} G}} \hat{\xi}_{0} \hat{\zeta}_{0}=-\kappa
\end{aligned}
$$

Compatibility requires

$$
\frac{2 \hat{\xi}_{0} \hat{\zeta}_{0}}{\sqrt{\operatorname{Det} G}}=1-s^{2}, \quad \kappa=s
$$

At the same time

$$
\left(\xi \tau a^{\dagger}\right)\left(\zeta C^{\prime} a^{\dagger}\right) \rightarrow-\hat{\xi}_{0} \hat{\zeta}_{0}\left(\left(a_{0}^{24 \dagger}\right)^{2}+\left(a_{0}^{25 \dagger}\right)^{2}\right)=-\frac{\hat{\xi}_{0} \hat{\zeta}_{0}}{\sqrt{\operatorname{Det} G}} a_{0}^{\alpha \dagger} G_{\alpha \beta} a_{0}^{\beta \dagger}
$$

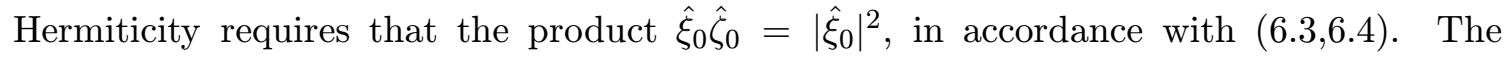
solutions found in this way can be referred to as the factorized solutions, since, as will become clear in a moment, they realize the factorization of the star product into the Moyal $\star$ product and Witten's $*$ product. In order to be able to compute $\left\langle x \mid \Lambda_{n}\right\rangle$ in the field theory limit, we have to evaluate first

$$
\begin{aligned}
\left\langle x\left|\left(a_{0}^{\alpha \dagger} G_{\alpha \beta} a_{0}^{\beta \dagger}\right)^{k} e^{-\frac{s}{2} a_{0}^{\alpha \dagger} G_{\alpha \beta} a_{0}^{\beta \dagger}}\right| \Omega_{b, \theta}\right\rangle & =(-2)^{k} \frac{d^{k}}{d s^{k}}\left(\left\langle x\left|e^{-\frac{s}{2} a_{0}^{\alpha \dagger} G_{\alpha \beta} a_{0}^{\beta \dagger}}\right| \Omega_{b, \theta}\right\rangle\right) \\
& =(-2)^{k} \frac{d^{k}}{d s^{k}}\left(\sqrt{\frac{2 \sqrt{\operatorname{Det} G}}{b \pi}} \frac{1}{1+s} e^{-\frac{1-s}{1+s} \frac{1}{b} x^{\alpha} G_{\alpha \beta} x^{\beta}}\right)
\end{aligned}
$$

An explicit calculation gives

$$
\begin{aligned}
& \frac{d^{k}}{d s^{k}}\left(\frac{1}{1+s} e^{-\frac{1-s}{1+s} \frac{1}{b} x^{\alpha} x^{\beta} G_{\alpha \beta}}\right)= \\
& \quad=\sum_{l=0}^{k} \sum_{j=0}^{k-l} \frac{(-1)^{k+j}}{(1-s)^{j}(1+s)^{k+1}} \frac{k !}{j !}\left(\begin{array}{c}
k-l-1 \\
j-1
\end{array}\right)\langle x, x\rangle^{j} e^{-\frac{1}{2}\langle x, x\rangle}
\end{aligned}
$$


where it must be understood that, by definition, the binomial coefficient $\left(\begin{array}{l}-1 \\ -1\end{array}\right)$ equals 1 . Moreover we have set

$$
\langle x, x\rangle=\frac{1}{a b} x^{\alpha} G_{\alpha \beta} x^{\beta}=\frac{2 r^{2}}{\theta}
$$

with $r^{2}=x^{\alpha} x^{\beta} \delta_{\alpha \beta}$.

Now, inserting (1) $\left(\Lambda_{n}\right\rangle$, we obtain after suitably reshuffling the indices:

$$
\begin{aligned}
\langle x|(-\kappa)^{n} L_{n}\left(\frac{\mathbf{x}}{\kappa}\right) e^{-\frac{1}{2} s a_{0}^{\alpha \dagger} G_{\alpha \beta} a_{0}^{\beta \dagger}\left|\Omega_{b, \theta}\right\rangle} \rightarrow & \left\langle x\left|(-s)^{n} L_{n}\left(-\frac{1-s^{2}}{2 s} a_{0}^{\alpha \dagger} G_{\alpha \beta} a_{0}^{\beta \dagger}\right) e^{-\frac{1}{2} s a_{0}^{\alpha \dagger} G_{\alpha \beta} a_{0}^{\beta \dagger}}\right| \Omega_{b, \theta}\right\rangle \\
= & \frac{(-s)^{n}}{(1+s)} \sum_{j=0}^{n} \sum_{k=j}^{n} \sum_{l=j}^{k}\left(\begin{array}{c}
n \\
k
\end{array}\right)\left(\begin{array}{c}
l-1 \\
j-1
\end{array}\right) \frac{1}{j !} \frac{(1-s)^{k}}{(1+s)^{j} s^{k}} \\
& (-1)^{j}\langle x, x\rangle^{j} e^{-\frac{1}{2}\langle x, x\rangle} \sqrt{\frac{2 \sqrt{\operatorname{Det} G}}{b \pi}}
\end{aligned}
$$

The expression can be evaluated as follows. First one uses the result

$$
\sum_{l=j}^{k}\left(\begin{array}{l}
l-1 \\
j-1
\end{array}\right)=\left(\begin{array}{l}
k \\
j
\end{array}\right)
$$

Inserting this into $(6.10 \mathrm{j})$ one is left with the following summation, which contains an evident binomial expansion,

$$
\sum_{k=j}^{n}\left(\begin{array}{l}
n \\
k
\end{array}\right)\left(\begin{array}{l}
k \\
j
\end{array}\right)\left(\frac{1-s}{s}\right)^{k}=\left(\begin{array}{l}
n \\
j
\end{array}\right) \frac{(1-s)^{j}}{s^{n}}
$$

Replacing this result into $(6 . \overline{10})$ we obtain

$$
\left\langle x\left|(-\kappa)^{n} L_{n}\left(\frac{\mathbf{x}}{\kappa}\right) e^{-\frac{1}{2} s a_{0}^{\alpha \dagger} G_{\alpha \beta} a_{0}^{\beta \dagger}}\right| \Omega_{b, \theta}\right\rangle \rightarrow \frac{2|a|+1}{4|a|} \sqrt{\frac{2 \sqrt{\operatorname{Det} G}}{b \pi}}(-1)^{n} \sum_{j=0}^{n}\left(\begin{array}{c}
n \\
j
\end{array}\right) \frac{1}{j !}\left(-\frac{2 r^{2}}{\theta}\right)^{j} e^{-\frac{r^{2}}{\theta}}
$$

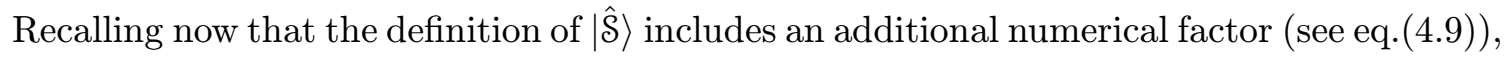
we finally obtain

$$
\begin{aligned}
\left\langle x \mid \Lambda_{n}\right\rangle \rightarrow\left\langle x \mid \hat{\Lambda}_{n}\right\rangle & =\frac{1}{\pi}(-1)^{n} \sum_{j=0}^{n}\left(\begin{array}{c}
n \\
j
\end{array}\right) \frac{1}{j !}\left(-\frac{2 r^{2}}{\theta}\right)^{j} e^{-\frac{r^{2}}{\theta}}|\Xi\rangle \\
& =\frac{1}{\pi}(-1)^{n} L_{n}\left(\frac{2 r^{2}}{\theta}\right) e^{-\frac{r^{2}}{\theta}}|\Xi\rangle
\end{aligned}
$$

as announced in the introduction. The coefficient in front of the sliver $|\Xi\rangle$ is the $n-t h$ GMS solution. Strictly speaking there is a discrepancy between these coefficients and the corresponding GMS soliton, given by the normalizations which differ by a factor of $2 \pi$. This can be traced back to the traditional normalizations used for the eigenstates $|x\rangle$ and $|p\rangle$ in the SFT theory context and in the Moyal context, respectively. This discrepancy can be easily dealt with a simple redefinition. 


\section{VSFT star product and Moyal product}

In the previous section we have shown that the low energy limit of $\left\langle x \mid \hat{\Lambda}_{n}\right\rangle$ factorizes into

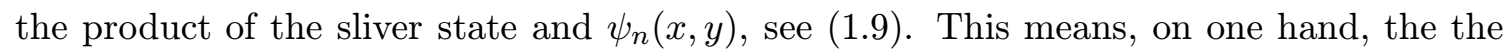
GMS solitons are the low energy remnants of corresponding D-branes in VSFT, and, on the other hand, that, for this type of solutions, the VSFT star product factorizes into Witten's star product and the Moyal $\star$ product. But, actually, much more can be said about the correspondence between the states $\left|\hat{\Lambda}_{n}\right\rangle$ and the solitons of noncommutative field theories with polynomial interaction.

We recall from the introduction that the latter are very elegantly constructed in terms of harmonic oscillators eigenstates $|n\rangle$. In particular the $\psi_{n}(x, y)$ solutions correspond to projectors $P_{n}=|n\rangle\langle n|$, via the Weyl transform. The correspondence is such that the operator product in the Hilbert space correspond to the Moyal product in $(x, y)$ space. Therefore we can formalize the following correspondence

$$
\begin{aligned}
\left|\Lambda_{n}\right\rangle & \longleftrightarrow P_{n} \longleftrightarrow \psi_{n}(x, y) \\
\left|\Lambda_{n}\right\rangle *\left|\Lambda_{n^{\prime}}\right\rangle & \longleftrightarrow P_{n} P_{n^{\prime}} \longleftrightarrow \psi_{n} \star \psi_{n^{\prime}}
\end{aligned}
$$

where $\star$ denotes the Moyal product. Moreover

$$
\left\langle\Lambda_{n} \mid \Lambda_{n^{\prime}}\right\rangle \longleftrightarrow \operatorname{Tr}\left(P_{n} P_{n^{\prime}}\right) \longleftrightarrow \int d x d y \psi_{n}(x, y) \psi_{n^{\prime}}(x, y)
$$

up to normalization (see ( $1.12 i)$ ). This correspondence seems to indicate that the Laguerre polynomials hide a universal structure of these noncommutative algebras.

This parallelism can actually be pushed still further. In fact we can easily construct the correspondents of the operators $|n\rangle\langle m|$. Let us first define

$$
X=a^{\dagger} \tau \xi \quad Y=a^{\dagger} C^{\prime} \zeta
$$

so that $\mathbf{x}=X Y$. The definitions we are looking for are as follows

$$
\begin{aligned}
& \left|\Lambda_{n, m}\right\rangle=\sqrt{\frac{n !}{m !}}(-\kappa)^{n} Y^{m-n} L_{n}^{m-n}\left(\frac{\mathbf{x}}{\kappa}\right)\left|\mathcal{S}_{\perp}\right\rangle, \quad n \leq m \\
& \left|\Lambda_{n, m}\right\rangle=\sqrt{\frac{m !}{n !}}(-\kappa)^{m} X^{n-m} L_{m}^{n-m}\left(\frac{\mathbf{x}}{\kappa}\right)\left|\mathcal{S}_{\perp}\right\rangle, \quad n \geq m
\end{aligned}
$$

where $L_{n}^{m-n}(z)=\sum_{k=0}^{m}\left(\begin{array}{c}m \\ n-k\end{array}\right)(-z)^{k} / k$ !. With the same techniques as in the previous sections one can prove that

$$
\left|\Lambda_{n, m}\right\rangle *\left|\Lambda_{r, s}\right\rangle=\delta_{m, r}\left|\Lambda_{n, s}\right\rangle
$$

for all natural numbers $n, m, r, s$. It is clear that the previous states $\left|\Lambda_{n}\right\rangle$ coincide with $\left|\Lambda_{n, n}\right\rangle$. In view of $\left(\overline{7}_{\overline{7}} \cdot \overline{6}^{\prime}\right)$, we can extend the correspondence $\left(\overline{7}_{-1} \overline{1}_{1}\right)$ to $|n\rangle\left\langle m|\leftrightarrow| \Lambda_{n, m}\right\rangle$. Therefore, following [ [45in star algebra the solution generating technique, in the same way as in the harmonic oscillator Hilbert space $\mathcal{H}$. Naturally in this case we do not have any guarantee that all the projectors are recovered in this way. 


\section{A. Properties of the Neumann coefficients with nonzero $B$}

In this Appendix we derive the properties of the coefficients $\mathcal{V}_{M N}^{\alpha \beta, r s}$ which are essential for the later developments. These properties are parallel to those enjoyed by the ordinary

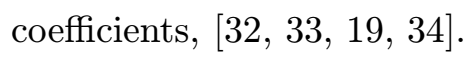

Let us quote first two straightforward properties of $\mathcal{V}_{M N}^{\alpha \beta, r s}$ :

- (i) they are symmetric under simultaneous exchange of the three couples of indices;

- (ii) they are endowed with the property of cyclicity in the $r, s$ indices, i.e. $\mathcal{V}^{r s}=$ $\mathcal{V}^{r+1, s+1}$, where $r, s=4$ is identified with $r, s=1$.

The first property is immediate. We pass immediately to the derivation of the latter. To this end we need the following representation of the coefficients $V_{0 n}^{r s}$, derived from [i32i]:

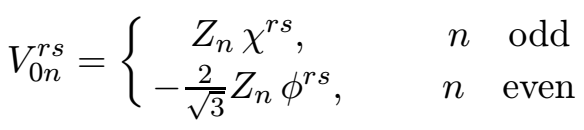

where

$$
Z_{n}=\sqrt{\frac{2}{3 n}} B_{0} A_{n}
$$

The numbers $B_{0}$ and $A_{n}$ were defined in ref.[32in. Notice that, since we have assumed $Z_{n}^{r s}=Z_{n}^{s r}$, we must have, by definition, $V_{0 n}^{r s}=V_{n 0}^{r s}$ for $n$ even and $V_{0 n}^{r s}=-V_{n 0}^{r s}$ for $n$ odd. Finally, for convenience, we introduce $Z_{0}=\sqrt{\frac{b}{3}}$.

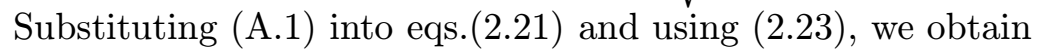

$$
\mathcal{V}_{N M}^{\alpha \beta, r s}=\left\{\begin{array}{ccc}
\mathcal{V}_{N M}^{\alpha \beta, r s}(\infty)-\frac{6 A^{-1}}{4 a^{2}+3} K_{\infty}^{\alpha \beta, r s} Z_{N} Z_{M}, & N+M & \text { even } \\
\mathcal{V}_{N M}^{\alpha \beta, r s}(\infty)+\frac{\sqrt{3} A^{-1}}{4 a^{2}+3} H_{\infty}^{\alpha \beta, r s}(-1)^{N} Z_{N} Z_{M}, & N+M & \text { odd }
\end{array}\right.
$$

In these equations

$$
\begin{aligned}
& K_{\infty}^{\alpha \beta, r s}=G^{\alpha \beta} \phi^{r s}-i a \epsilon^{\alpha \beta} \chi^{r s} \\
& H_{\infty}^{\alpha \beta, r s}=3 G^{\alpha \beta} \chi^{r s}+4 i a \epsilon^{\alpha \beta} \phi^{r s}
\end{aligned}
$$

and $\mathcal{V}_{N M}^{\alpha \beta, r s}(\infty)$ is

$$
\begin{aligned}
& \mathcal{V}_{00}^{\alpha \beta, r s}(\infty)=G^{\alpha \beta} \delta^{r s} \\
& \mathcal{V}_{0 m}^{\alpha \beta, r s}(\infty)=0 \\
& \mathcal{V}_{n m}^{\alpha \beta, r s}(\infty)=G^{\alpha \beta} V_{n m}^{r s}
\end{aligned}
$$

The coefficients $V_{n m}^{r s}$ are the same as in ref. [3] $\left.\overline{4} \overline{4}\right]$ for $n, m \geq 1$.

Next we introduce the third root of unity $\omega=e^{i \frac{2 \pi}{3}}$ and notice that

$$
\phi^{r s}=\frac{1}{2}\left(\omega^{r-s}+\omega^{s-r}\right), \quad \chi^{r s}=\frac{i}{\sqrt{3}}\left(\omega^{r-s}-\omega^{s-r}\right),
$$


Inserting these relations into ( $\left(A^{A} . \overline{3}_{1}^{\prime}\right)$ and rearranging the terms we find the basic relation

$$
\nu_{N M}^{\alpha \beta, r s}=\frac{1}{3}\left(C_{N M}^{\prime} G^{\alpha \beta}+\omega^{s-r} \mathcal{U}_{N M}^{\alpha \beta}+\omega^{r-s} \overline{\mathcal{U}}_{N M}^{\alpha \beta}\right)
$$

where

$$
\mathcal{U}_{N M}^{\alpha \beta}=\left\{\begin{array}{ccc}
G^{\alpha \beta} \mathfrak{U}_{N M}(\infty)+R^{\alpha \beta} Z_{N} Z_{M}, & N+M & \text { even } \\
G^{\alpha \beta} \mathcal{U}_{N M}(\infty)+i R^{\alpha \beta}(-1)^{N} Z_{N} Z_{M}, & N+M & \text { odd }
\end{array}\right.
$$

Moreover

$$
\overline{\mathcal{U}}^{\alpha \beta}=\left(\mathcal{U}^{\beta \alpha}\right)^{*}
$$

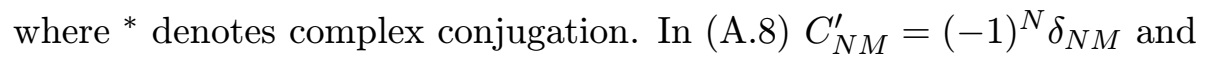

$$
R^{\alpha \beta}=\frac{6 A^{-1}}{4 a^{2}+3}\left(-\frac{3}{2} G^{\alpha \beta}+\sqrt{3} a \epsilon^{\alpha \beta}\right)
$$

Moreover

$$
\begin{aligned}
& \mathcal{U}_{00}^{\alpha \beta}(\infty)=G^{\alpha \beta}, \quad \mathcal{U}_{0 n}^{\alpha \beta}=0 \\
& \mathcal{U}_{n m}^{\alpha \beta}(\infty)=G^{\alpha \beta} U_{n m}
\end{aligned}
$$

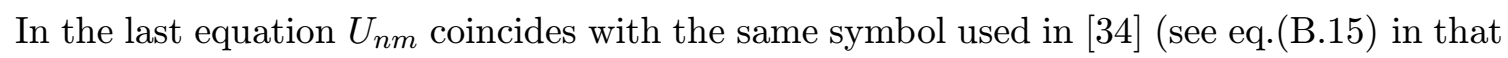
reference).

Let us discuss the properties of $\mathcal{U}$. Since

$$
\left(\mathcal{U}_{N M}^{\alpha \beta}\right)^{*}=\left\{\begin{array}{ccc}
\mathcal{U}_{N M}^{\alpha \beta}, & N+M & \text { even } \\
-U_{N M}^{\alpha \beta}, & N+M & \text { odd }
\end{array}\right.
$$

it is easy to prove the following properties

$$
\left(\mathcal{U}^{\alpha \beta}\right)^{*}=C^{\prime} \mathcal{U}^{\alpha \beta} C^{\prime}\left(\mathcal{U}^{\alpha \beta}\right)^{\dagger}=\left(\mathcal{U}^{\alpha \beta}\right)^{* T}=\left(C^{\prime} \mathfrak{U}^{\alpha \beta} C^{\prime}\right)^{T}=\mathcal{U}^{\alpha \beta}
$$

Finally, if tilde denotes transposition in the indices $\alpha, \beta$, it is possible to prove that (see Appendix A of [4 $46 \overline{6}]$ )

$$
(\mathcal{U} \tilde{U})_{N M}^{\alpha \beta}=(\tilde{\mathcal{U}})_{N M}^{\alpha \beta}=G^{\alpha \beta} \delta_{N M}+\left(R G+G \tilde{R}+\frac{2}{3} A R \tilde{R}\right) Z_{N} Z_{M}
$$

Now, remembering that $\epsilon^{\alpha \gamma} \epsilon_{\gamma}{ }^{\beta}=-G^{\alpha \beta}$, it is elementary to prove that

$$
R G+G \tilde{R}+\frac{2}{3} A R \tilde{R}=0
$$

Therefore, finally,

$$
(\mathcal{U} \tilde{U})_{N M}^{\alpha \beta}=(\tilde{\mathcal{U}})_{N M}^{\alpha \beta}=G^{\alpha \beta} \delta_{N M}
$$

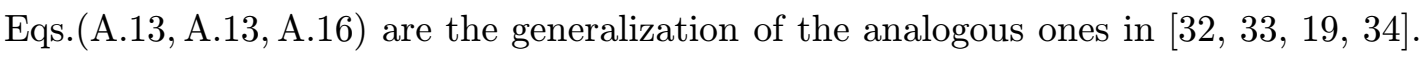
Using in particular ( $\left(A-16^{\prime}\right)$, it is easy to prove that

$$
\left[C^{\prime} \mathcal{V}^{r s}, C^{\prime} \mathcal{V}^{r^{\prime} s^{\prime}}\right]=0
$$

This follows from

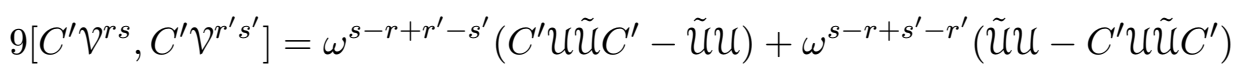

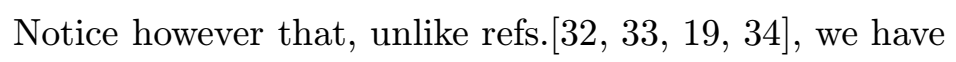

$$
C^{\prime} \mathcal{V}^{r s}=\tilde{\mathcal{V}}^{s r} C^{\prime}
$$


B. Proof of $\left|\Lambda_{n}\right\rangle *\left|\Lambda_{m}\right\rangle=\delta_{n, m}\left|\Lambda_{n}\right\rangle$

The star product $\left|\Lambda_{n}\right\rangle *\left|\Lambda_{n^{\prime}}\right\rangle$ can be evaluated by using the explicit expression of the Laguerre polynomials

$$
\left|\Lambda_{n}\right\rangle *\left|\Lambda_{n^{\prime}}\right\rangle=\left((-\kappa)^{n} \sum_{k=0}^{n}\left(\begin{array}{l}
n \\
k
\end{array}\right) \frac{(-\mathbf{x} / \kappa)^{k}}{k !}\left|\mathcal{S}_{\perp}\right\rangle\right) *\left((-\kappa)^{n^{\prime}} \sum_{p=0}^{n^{\prime}}\left(\begin{array}{c}
n^{\prime} \\
p
\end{array}\right) \frac{(-\mathbf{x} / \kappa)^{p}}{p !}\left|\mathcal{S}_{\perp}\right\rangle\right)(\mathrm{B}
$$

Therefore we need to compute $\left(\mathbf{x}^{k}\left|\mathcal{S}_{\perp}\right\rangle\right) *\left(\mathbf{x}^{p}\left|\mathcal{S}_{\perp}\right\rangle\right)$. According to [1] $\left.\overline{2} \overline{1}\right]$, this is given by

$$
\begin{aligned}
\left(\mathbf{x}^{k}\left|\mathcal{S}_{\perp}\right\rangle\right) *\left(\mathbf{x}^{p}\left|\mathcal{S}_{\perp}\right\rangle\right)= & \left(\xi \tau C^{\prime}\right)_{l_{1}}^{\alpha_{1}} \ldots\left(\xi \tau C^{\prime}\right)_{l_{k}}^{\alpha_{k}} \zeta_{j_{1}}^{\beta_{1}} \ldots \zeta_{j_{k}}^{\beta_{k}} \frac{\partial}{\partial \mu_{l_{1}}^{\alpha_{1}}} \cdots \frac{\partial}{\partial \mu_{l_{k}}^{\alpha_{k}}} \frac{\partial}{\partial \mu_{j_{1}}^{\beta_{1}}} \cdots \frac{\partial}{\partial \mu_{j_{k}}^{\beta_{k}}} \\
& \cdot\left(\xi \tau C^{\prime}\right)_{\bar{l}_{1}}^{\bar{\alpha}_{1}} \ldots\left(\xi \tau C^{\prime}\right)_{\bar{l}_{p}}^{\bar{\alpha}_{p}} \zeta_{\bar{j}_{1}}^{\bar{\beta}_{1}} \ldots \zeta_{\bar{j}_{p}}^{\bar{\beta}_{p}} \frac{\partial}{\partial \bar{\mu}_{\bar{l}_{1}}^{\bar{\alpha}_{1}}} \ldots \frac{\partial}{\partial \bar{\mu}_{\bar{l}_{p}}^{\bar{\alpha}_{p}}} \frac{\partial}{\partial \bar{\mu}_{\bar{j}_{1}}^{\bar{\beta}_{1}}} \cdots \frac{\partial}{\partial \bar{\mu}_{\bar{j}_{p}}^{\bar{\beta}_{p}}} \\
& \left.\cdot \exp \left(-\chi^{T} \mathcal{K}^{-1} M-\frac{1}{2} M^{T} \mathcal{V} \mathcal{K}^{-1} M\right)\left|\mathcal{S}_{\perp}\right\rangle\right)\left.\right|_{\mu=\bar{\mu}=0}
\end{aligned}
$$

where

$$
\mathcal{K}=\mathbb{I}-\mathcal{T} X, \quad \mathcal{V}=\left(\begin{array}{ll}
\mathcal{V}^{11} & \mathcal{V}^{12} \\
\mathcal{V}^{21} & \mathcal{V}^{22}
\end{array}\right)
$$

and

$$
M=\left(\begin{array}{c}
\mu \\
\bar{\mu}
\end{array}\right), \quad \chi^{T}=\left(a^{\dagger} \nu^{12}, a^{\dagger} \nu^{21}\right), \quad \chi^{T} \mathcal{K}^{-1} M=a^{\dagger} C^{\prime}\left(\rho_{1} \mu+\rho_{2} \bar{\mu}\right)
$$

Now, we avail ourselves of the following identities

$$
\begin{aligned}
& \xi^{T}\left(\mathcal{V K} \mathcal{K}^{-1}\right)_{\alpha \alpha} \zeta=\xi^{T} \tau C^{\prime}\left(\mathcal{V K}{ }^{-1}\right)_{\alpha \alpha} \tau C^{\prime} \zeta=\xi^{T} C^{\prime} \frac{\mathcal{T}}{\mathbb{I}-\mathcal{T}^{2}} \zeta=0 \\
& \xi^{T} \tau C^{\prime}\left(\mathcal{V K} \mathcal{K}^{-1}\right)_{\alpha \alpha} \zeta=\xi^{T}\left(\mathcal{V} \mathcal{K}^{-1}\right)_{\alpha \alpha} \tau C^{\prime} \zeta=\xi^{T} \tau \frac{\mathcal{T}}{\mathbb{I}-\mathcal{T}^{2}} \zeta=-\kappa
\end{aligned}
$$

for $\alpha=1,2$, and

$$
\begin{aligned}
& \xi^{T}\left(\mathcal{V} \mathcal{K}^{-1}\right)_{12} \zeta=\xi^{T} \tau C^{\prime}\left(\mathcal{V} \mathcal{K}^{-1}\right)_{21} \tau C^{\prime} \zeta=-\xi^{T} C^{\prime} \frac{\mathcal{T}}{\mathbb{I}-\mathcal{T}^{2}} \zeta=0 \\
& \xi^{T}\left(\mathcal{V K} \mathcal{K}^{-1}\right)_{21} \zeta=\xi^{T} \tau C^{\prime}\left(\mathcal{V} \mathcal{K}^{-1}\right)_{12} \tau C^{\prime} \zeta=\xi^{T} C^{\prime} \frac{1}{\mathbb{I}-\mathcal{T}^{2}} \zeta=0 \\
& \xi^{T}\left(\mathcal{V} \mathcal{K}^{-1}\right)_{12} \tau C^{\prime} \zeta=\xi^{T} \tau C^{\prime}\left(\mathcal{V} \mathcal{K}^{-1}\right)_{21} \zeta=\xi^{T} \tau \frac{1}{\mathbb{I}-\mathcal{T}^{2}} \zeta=-1 \\
& \xi^{T} \tau C^{\prime}\left(\mathcal{V} \mathcal{K}^{-1}\right)_{12} \zeta=\xi^{T}\left(\mathcal{V} \mathcal{K}^{-1}\right)_{21} \tau C^{\prime} \zeta=-\xi^{T} \tau \frac{\mathcal{T}}{\mathbb{I}-\mathcal{T}^{2}} \zeta=\kappa
\end{aligned}
$$

Moreover

$$
\begin{aligned}
& \left(\chi^{T} \mathcal{K}^{-1}\right)_{1} \xi=0, \quad\left(\chi^{T} \mathcal{K}^{-1}\right)_{1} \tau C^{\prime} \xi=a^{\dagger} \tau \xi \\
& \left(\chi^{T} \mathcal{K}^{-1}\right)_{2} \xi=a^{\dagger} C^{\prime} \xi, \quad\left(\chi^{T} \mathcal{K}^{-1}\right)_{2} \tau C^{\prime} \xi=0
\end{aligned}
$$


with analogous equations for $\zeta$.

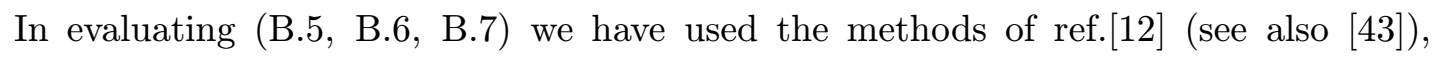

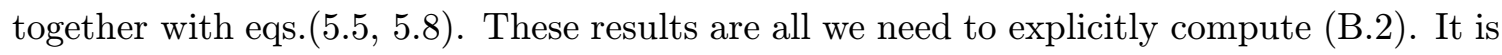
easy to see that the computation can be mapped to a rather simple combinatorial problem, introducing generic variables $x, y, \bar{x}, \bar{y}$, and making the following formal replacements:

$$
\begin{aligned}
& A \equiv \chi^{T} \mathcal{K}^{-1} M \longrightarrow x\left(a^{\dagger} \tau \xi\right)+\bar{y}\left(a^{\dagger} C^{\prime} \zeta\right), \\
& B \equiv M^{T} \mathcal{V} \mathcal{K}^{-1} M \longrightarrow(-\kappa x y+\kappa x \bar{y}-\bar{x} y-\kappa \bar{x} \bar{y})
\end{aligned}
$$

and

$$
\left(\tau C^{\prime} \xi\right)_{l}^{\alpha} \frac{\partial}{\partial \mu_{l}^{\alpha}}=\partial_{x}, \quad \zeta_{j}^{\beta} \frac{\partial}{\partial \mu_{j}^{\beta}}=\partial_{y}, \quad\left(\tau C^{\prime} \xi\right)_{\bar{l}}^{\bar{\alpha}} \frac{\partial}{\partial \bar{\mu}_{\bar{l}}^{\bar{\alpha}}}=\partial_{\bar{x}}, \quad \zeta_{\bar{j}}^{\bar{\beta}} \frac{\partial}{\partial \bar{\mu}_{\bar{j}}^{\bar{\beta}}}=\partial_{\bar{y}}
$$

Then (B. $\overline{2}$ - is equivalent to

$$
\left.\partial_{x}^{k} \partial_{y}^{k} \partial_{\bar{x}}^{p} \partial_{\bar{y}}^{p} e^{-A-\frac{1}{2} B}\right|_{x=\bar{x}=y=\bar{y}=0}
$$

This in turn can be easily calculated and gives

$$
\sum_{m=0}^{[p, k]} \mathbf{x}^{m} \frac{k ! p !}{m !} \sum_{l=m}^{[p, k]}(-1)^{l+m}\left(\begin{array}{l}
k \\
l
\end{array}\right)\left(\begin{array}{l}
p \\
l
\end{array}\right)\left(\begin{array}{c}
l \\
m
\end{array}\right) \kappa^{p+k-l-2 m}
$$

where $[n, m]$ stands for the minimum between $n$ and $m$. Now we insert this back into the original equation $\left(\bar{B}_{-} \cdot \overline{1} \bar{i}\right)$, we find

$$
\begin{aligned}
& \left|\Lambda_{n}\right\rangle *\left|\Lambda_{n^{\prime}}\right\rangle=\sum_{k=0}^{n} \sum_{p=0}^{n^{\prime}} \sum_{m=0}^{[p, k]} \sum_{l=0}^{[p-m, k-m]} \frac{(-1)^{p+k+l}}{m !} \\
& \cdot \kappa^{n+n^{\prime}-l-2 m}\left(\begin{array}{c}
n \\
k
\end{array}\right)\left(\begin{array}{c}
n^{\prime} \\
p
\end{array}\right)\left(\begin{array}{c}
k \\
m
\end{array}\right)\left(\begin{array}{c}
k-m \\
l
\end{array}\right)\left(\begin{array}{c}
p \\
l+m
\end{array}\right) \mathbf{x}^{m}\left|\mathcal{S}_{\perp}\right\rangle
\end{aligned}
$$

In order to evaluate these summations we split them, we replace $l \rightarrow l+m$ and finally we obtain

$$
\begin{aligned}
\left|\Lambda_{n}\right\rangle *\left|\Lambda_{n^{\prime}}\right\rangle= & \sum_{k=0}^{n} \sum_{m=0}^{k} \sum_{l=m}^{k} \sum_{p=l}^{n^{\prime}} \frac{(-1)^{p+k+l+m}}{m !} \\
& \cdot \kappa^{n+n^{\prime}-l-m}\left(\begin{array}{c}
n \\
k
\end{array}\right)\left(\begin{array}{c}
n^{\prime} \\
p
\end{array}\right)\left(\begin{array}{c}
k \\
m
\end{array}\right)\left(\begin{array}{c}
k-m \\
l-m
\end{array}\right)\left(\begin{array}{c}
p \\
l
\end{array}\right) \mathbf{x}^{m}\left|\mathcal{S}_{\perp}\right\rangle
\end{aligned}
$$

Now

$$
\sum_{p=l}^{n^{\prime}}(-1)^{p+l}\left(\begin{array}{c}
n^{\prime} \\
p
\end{array}\right)\left(\begin{array}{c}
p \\
l
\end{array}\right)=\left(\begin{array}{c}
n^{\prime} \\
l
\end{array}\right) \sum_{p=0}^{n^{\prime}-l}(-1)^{p}\left(\begin{array}{c}
n^{\prime}-l \\
p
\end{array}\right)=\left(\begin{array}{c}
n^{\prime} \\
l
\end{array}\right)(1-1)^{n^{\prime}-l}
$$

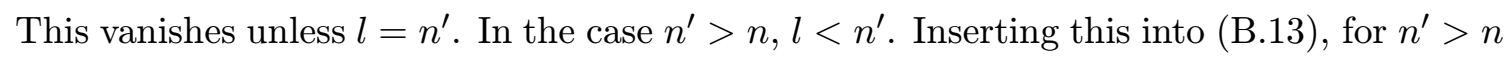
we get 0 . 
In the case $n=n^{\prime}, l$ can take the value $n^{\prime}$. This corresponds to the case $k=p=l=$ $n=n^{\prime}$ in eq.('(B).13is). The result is easily derived

$$
\left|\Lambda_{n}\right\rangle *\left|\Lambda_{n}\right\rangle=\sum_{m=0}^{n} \frac{(-1)^{n+m}}{m !}\left(\begin{array}{c}
n \\
m
\end{array}\right) \kappa^{n-m} \mathbf{x}^{m}\left|\mathcal{S}_{\perp}\right\rangle=(-\kappa)^{n} L_{n}\left(\frac{\mathbf{x}}{\kappa}\right)\left|\mathcal{S}_{\perp}\right\rangle=\left|\Lambda_{n}\right\rangle
$$

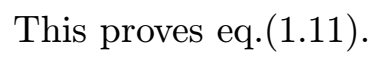

\section{Acknowledgments}

This research was supported by the Italian MIUR under the program "Teoria dei Campi, Superstringhe e Gravità".

\section{References}

[1] E.Witten, Noncommutative Geometry and String Field Theory, Nucl.Phys. B268 (1986) 253.

[2] A.Sen Descent Relations among Bosonic D-Branes, Int.J.Mod.Phys. A14 (1999) 4061, [hep-th/9902105]. Tachyon Condensation on the Brane Antibrane System JHEP 9808 (1998) 012 , hhep-th/9805170i. BPS D-branes on Non-supersymmetric Cycles, JHEP 9812 (1998) 021, hep-th/9812031!

[3] L.Rastelli, A.Sen and B.Zwiebach, String field theory around the tachyon vacuum, Adv. Theor. Math. Phys. 5 (2002) 353 [hep-th/00122511.

[4] D.Gaiotto, L.Rastelli, A.Sen and B.Zwiebach, Ghost Structure and Closed Strings in Vacuum String Field Theory, [hep-th/0111129i].

[5] H.Hata and H.Kogetsu Higher Level Open String States from Vacuum String Field Theory, [hep-th/0208067! .

[6] H.Hata and T.Kawano, Open string states around a classical solution in vacuum string field theory, JHEP 0111 (2001) 038 [hep-th/0108150i].

[7] K.Okuyama, Siegel Gauge in Vacuum String Field Theory, JHEP 0201 (2002) 043 [hep-th/011 1087.].

[8] K.Okuyama, Ghost Kinetic Operator of Vacuum String Field Theory, JHEP 0201 (2002) 027 hep-th/0201015.

[9] L.Rastelli, A.Sen and B.Zwiebach, A note on a Proposal for the Tachyon State in Vacuum String Field Theory, JHEP 0202 (2002) 034 [hep-th/0111153.

[10] L.Rastelli, A.Sen and B.Zwiebach, Star Algebra Spectroscopy, JHEP 0203 (2002) 029 [hep-th/0111281].

[11] I.Kishimoto, Some properties of string field algebra, JHEP 0112 (2001) 007 hep-th/0110124.

[12] L.Rastelli, A.Sen and B.Zwiebach, Half-strings, Projectors, and Multiple D-branes in Vacuum String Field Theory, JHEP 0111 (2001) 035 [hep-th/0105058].

[13] D.J.Gross and W.Taylor, Split string field theory. I JHEP 0108 (2001) 009 [hep-th/0105059], D.J.Gross and W.Taylor, Split string field theory. II JHEP 0108 (2001) $010[$ hep-th/0106036i. 
[14] T.Kawano and K.Okuyama, Open String Fields as Matrices, JHEP 0106 (2001) 061 [hep-th/0105129].

[15] N.Moeller, Some exact results on the matter star-product in the half-string formalism, JHEP 0201 (2002) 019 [hep-th/0110204].

[16] J.R.David, Excitations on wedge states and on the sliver, JHEP 0107 (2001) 024 hep-th/0105184.

[17] P.Mukhopadhyay, Oscillator representation of the BCFT construction of D-branes in vacuum string field theory, JHEP 0112 (2001) 025 [hep-th/0110136il.

[18] M.Schnabl, Wedge states in string field theory [hep-th/0201095]. Anomalous reparametrizations and butterfly states in string field theory, [hep-th/0202139i].

[19] V.A.Kostelecky and R.Potting, Analytical construction of a nonperturbative vacuum for the open bosonic string, Phys. Rev. D 63 (2001) 046007 [hep-th/0008252i].

[20] J. A. Harvey, Komaba lectures on noncommutative solitons and D-branes, [hep-th/0102076].

[21] E.Witten, Noncommutative Tachyons and String Field Theory, [hep-th/ōō6-711].

[22] M.Schnabl, String field theory at large B-field and noncommutative geometry, JHEP 0011, (2000) $031[$ [hep-th/0010034].

[23] F.Sugino, Witten's Open String Field Theory in Constant B-field Background, JHEP 0003, (2000) 017 [hep-th/9912254].

[24] T.Kawano and T.Takahashi, Open String Field Theory on Noncommutative Space, Prog. Theor. Phys. 104 (2000) 459 [hep-th/9912274].

[25] P. Matlock, R. C. Rashkov, K. S. Viswanathan and Y. Yang, Dp-brane tension from tachyons and B-field in Vacuum String Field Theory, Phys. Rev. D 66 (2002) 026004 $[$ hep-th/0203071].

[26] I.Bars, Map of Witten's * to Moyal's * Phys.Lett. B517 (2001) 436. [hep-th/0106157].

[27] M.R. Douglas, H. Liu, G. Moore, B. Zwiebach, Open String Star as a Continuous Moyal Product, JHEP 0204 (2002) 022 [hep-th/0202087].

[28] F.Bayen, M.Flato, C.Fronsdal, A.Lichnerowicz, D.Sternheimer, Deformation Theory of Quantization. I, II, Ann.Phys. 111 (1978) 61, 112.

[29] B. Feng, Y.-H. He and N. Moeller, Zeeman Spectroscopy of the Star Algebra, JHEP 0205 (2002) 041 [hep-th/0203175].

[30] B. Chen, F. L. Lin, Star Spectroscopy in the Constant B field Background, Nucl. Phys. B 637 (2002) 199 [hep-th/0203204]. D-branes as GMS solitons in vacuum string field theory [hep-th/0204233].

[31] D. M. Belov, Diagonal representation of open string star and Moyal product, [hep-th/0204164].

[32] D.J.Gross and A.Jevicki, Operator Formulation of Interacting String Field Theory, Nucl.Phys. B283 (1987) 1.

[33] D.J.Gross and A.Jevicki, Operator Formulation of Interacting String Field Theory, 2, Nucl.Phys. B287 (1987) 225. 
[34] L.Rastelli, A.Sen and B.Zwiebach, Classical solutions in string field theory around the tachyon vacuum, Adv. Theor. Math. Phys. 5 (2002) 393 [hep-th/0102112].

[35] N.Seiberg and E.Witten, String Theory and Noncommutative Geometry, JHEP 9909, (1999)

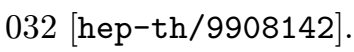

[36] T.Okuda, The Equality of Solutions in Vacuum String Field Theory, Nucl. Phys. B641 (2002) 393 [hep-th/0201149].

[37] E.Fuchs, M.Kroyter and A.Marcus, Squeezed States Projectors in String Field Theory, JHEP 0209 (2002) 022 [hep-th/0207001].

[38] D. Gaiotto, L. Rastelli, A. Sen and B. Zwiebach, Star Algebra Projectors, JHEP 0204 (2002) 060 [hep-th/0202151!.

[39] Y.Okawa, Open string states and D-brane tension form vacuum string field theory, JHEP 0207 (2002) 003 [hep-th/0204012].

[40] K.Okuyama, Ratio of Tensions from Vacuum String Field Theory, JHEP 0203 (2002) 050 [hep-th/0201136i].

[41] H.Hata and S.Moriyama, Observables as Twist Anomaly in Vacuum String Field Theory, JHEP 0201 (2002) 042 [hep-th/0111034.

[42] H.Hata and S.Moriyama, Reexamining Classical Solution and Tachyon Mode in Vacuum String Field Theory, hep-th/0206208․

[43] L. Bonora, D. Mamone and M. Salizzoni, Vacuum String Field Theory with B field, JHEP 0204 (2002) 020 [hep-th/0203188].

[44] G.Moore and W.Taylor The singular geometry of the sliver, JHEP 0201 (2002) 004 [hep-th/0111069.

[45] R. Gopakumar, S. Minwalla and A. Strominger, Noncommutative solitons, JHEP 0005 (2000) 020 [hep-th/0003160].

[46] L. Bonora, D. Mamone and M. Salizzoni, B field and squeezed states in Vacuum String Field Theory, Nucl. Phys. B630 (2002) 163 [hep-th/020̄1060].

[47] L. Bonora, D. Mamone and M. Salizzoni, Vacuum String Field Theory ancestors of the GMS solitons, [hep-th/0207044]. 